\title{
¿Why Do Precipitation Intensities Tend to Follow Gamma Distributions?
}

\author{
Cristian MartineZ-Villalobos and J. DAVID NeElin \\ Department of Atmospheric and Oceanic Sciences, University of California, Los Angeles, Los Angeles, California
}

(Manuscript received 27 November 2018, in final form 2 August 2019)

\begin{abstract}
The probability distribution of daily precipitation intensities, especially the probability of extremes, impacts a wide range of applications. In most regions this distribution decays slowly with size at first, approximately as a power law with an exponent between 0 and -1 , and then more sharply, for values larger than a characteristic cutoff scale. This cutoff is important because it limits the probability of extreme daily precipitation occurrences in current climate. There is a long history of representing daily precipitation using a gamma distribution-here we present theory for how daily precipitation distributions get their shape. Processes shaping daily precipitation distributions can be separated into nonprecipitating and precipitating regime effects, the former partially controlling how many times in a day it rains, and the latter set by single-storm accumulations. Using previously developed theory for precipitation accumulation distributions-which follow a sharper power-law range (exponent $<-1$ ) with a physically derived cutoff for large sizes - analytical expressions for daily precipitation distribution power-law exponent and cutoff are calculated as a function of key physical parameters. Precipitating and nonprecipitating regime processes both contribute to reducing the power-law range exponent for the daily precipitation distribution relative to the fundamental exponent set by accumulations. The daily precipitation distribution cutoff is set by the precipitating regime and scales with moisture availability, with important consequences for future distribution shifts under global warming. Similar results extend to different averaging periods, providing insight into how the precipitation intensity distribution evolves as a function of both underlying physical climate conditions and averaging time.
\end{abstract}

\section{Introduction}

The shape of the distribution of temporally averaged precipitation intensity has a long history of being represented by a gamma distribution since at least Thom (1958). For sufficiently short averaging periods (e.g., daily average intensities), the typical shape of the distribution has probability falling slowly over a few orders of magnitude, summarizing the wide range of precipitation intensities experienced in a given region. Is there a fundamental explanation for this behavior in terms of simple physical processes? The goals of this

¿ Denotes content that is immediately available upon publication as open access.

Supplemental information related to this paper is available at the Journals Online website: https://doi.org/10.1175/JAS-D-180343.s1.

\footnotetext{
Corresponding author: Cristian Martinez-Villalobos, cmartinezvil@ atmos.ucla.edu
}

paper are to (i) explain why temporally averaged distributions have a gamma-like distribution shape using a simple model based on the moisture equation [e.g., Neelin and Zeng 2000, their (2.2); Sobel and Maloney 2013, their (1)], and (ii) provide theory for how the gamma distribution parameters depend on physical processes and averaging interval (e.g., 3-hourly, daily, monthly precipitation). To anchor the discussion, we use daily precipitation statistics as the leading example, but results apply to other averaging intervals, as expanded in the final part of the paper.

A gamma distribution, as commonly used for describing temporally averaged precipitation statistics (Thom 1958; Ison et al. 1971; Katz 1977; Richardson 1981; Ropelewski et al. 1985; Wilks 1995; Groisman et al. 1999; Husak et al. 2007), is given by

$$
p_{x}=\frac{1}{\Gamma(k) \theta^{k}} x^{k-1} \exp \left(-\frac{x}{\theta}\right), \quad x>0,
$$

where $k$ is the shape parameter, $\theta$ the scale parameter, and $x$ represents temporally averaged precipitation. 


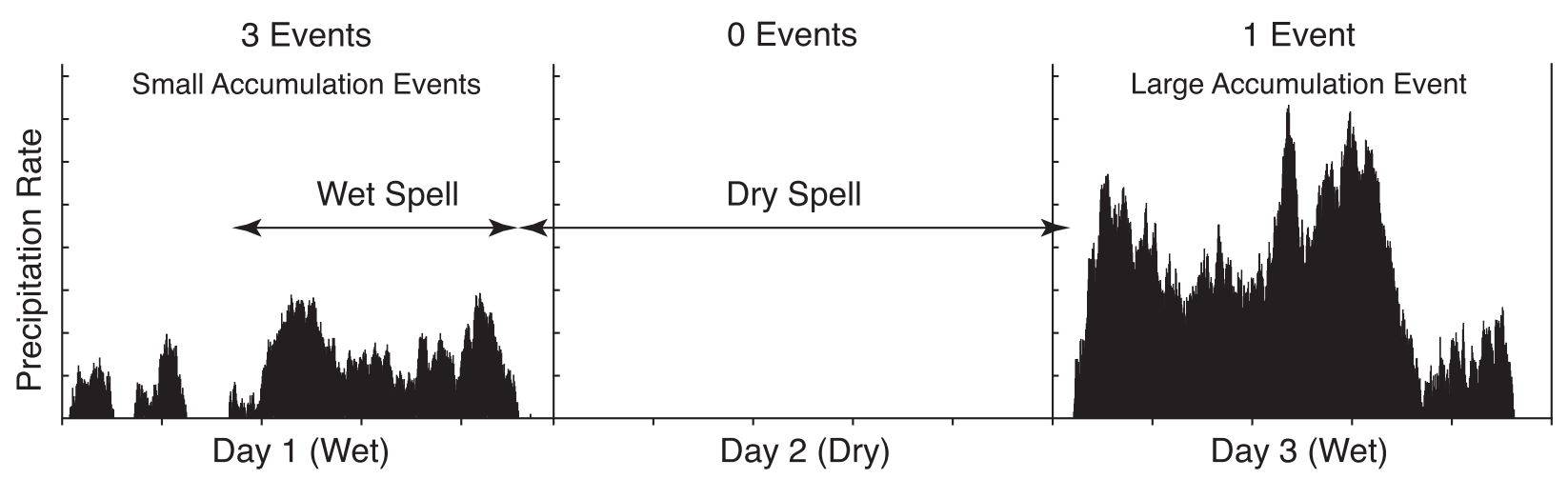

FIG. 1. Schematic showing an example of precipitation rate $\left(\mathrm{mm} \mathrm{h}^{-1}\right)$ for three consecutive days. The area under the curve from event onset and termination is the event accumulation $(\mathrm{mm})$. The total precipitation in a day is the summation of the accumulation events in that day. Events starting in one day and finishing the next are discussed in section 4.

For daily precipitation, the shape parameter controls the probability of light and moderate daily precipitation totals, while the scale parameter is a useful metric to track changes of the extremes (Groisman et al. 1999; Wilby and Wigley 2002; Watterson and Dix 2003; MartinezVillalobos and Neelin 2018b, hereafter MN18). As such, understanding the processes that control these parameters has important societal value.

In this paper we show that two main ingredients can be used to explain daily (or other temporal average) precipitation statistics:

(i) Knowledge of the distribution of precipitation accumulations, defined as the amount of precipitation integrated over an event from start to end of precipitation. The theory for this is outlined below.

(ii) Knowledge of the distribution of the number of times it precipitates within the temporal average scale of interest $t_{\mathrm{avg}}$. If $t_{\mathrm{avg}}$ is equal to 1 day this is referred to as the "daily number of events distribution," and similar for other $t_{\mathrm{avg}}$.

This is shown schematically in Fig. 1. Here the $y$ axis shows the instantaneous precipitation rate (for brevity "instantaneous rate" will be simply referred as "rate" in what follows), and the accumulation is the total amount from precipitation start to termination. There are three relatively small accumulation events on day 1 (wet day), zero events on day 2 (dry day), and one big accumulation event on day 3 (wet day), with the daily precipitation totals being the summation of the accumulations in each day. Here we show only three days, but in general there is a distribution of the number of times it precipitates in a day (daily number of events distribution), and a distribution of the total amount that it rains each time (accumulation distribution). The interplay between these two distributions shapes the resulting daily precipitation statistics.
In contrast to daily precipitation, the fundamental physical processes that shape accumulation distributions are reasonably well understood. This distribution by definition is only affected by processes occurring in the wet regime (from precipitation onset to termination). At the most fundamental, accumulation distributions can be understood using a few observationally constrained ingredients, based on the observed relationship between precipitation and column water vapor $q$ (Raymond 2000; Bretherton et al. 2004; Peters and Neelin 2006; Neelin et al. 2009; Muller et al. 2009). These include (i) a fundamental climate equation (the column water vapor equation), (ii) a threshold for precipitation onset, which reflects the observation that precipitation tend to start when column water vapor exceeds a certain threshold, given by convective instability or large-scale saturation, and (iii) a similarly defined threshold for precipitation termination. Using these simple ingredients, Stechmann and Neelin (2014, hereafter SN14) derive the fundamental shape of accumulation distributions, with qualitative success in explaining observed distributions (Peters et al. 2001, 2010; Deluca and Corral 2014; MN18). Importantly, the parameters of the SN14 derived accumulation distribution can be directly related to processes occurring in the wet regime, including a dependence of the probability of the most extreme accumulations on column water vapor (Neelin et al. 2017, hereafter N17), with important expected consequences under global warming.

The second important point is the distribution of the number of times it precipitates in a given time interval of interest. This number of events distribution encapsulates the effects of intermittency (Schleiss 2018) on the resulting time-averaged precipitation distributions. Here we show that this distribution depends on both the dry (i.e., between accumulation events; see Fig. 1) and 
wet (within precipitation accumulation events) regimes, as opposed to accumulation distributions that only depend on wet regime physics. This allows a conceptual separation between dry and wet regime effects on timeaveraged precipitation statistics.

In this paper we show how both these processes occurring in the wet and dry regimes combine to give shape to the observed precipitation distributions. Section 2 gives a brief overview of accumulations and daily precipitation distributions in observations. Section 3 presents two simple stochastic prototypes that are used to model the dry and wet regimes with simplified physics, and that provide a representation of daily (or longer averages) precipitation distributions. While reality is considerably more complex than these models, we argue that their simplicity is their main strength, as important insight into this problem can be gained that can be used to interpret observed temporally averaged precipitation distributions. Section 4 provides an explanation of why daily precipitation distributions are well fitted by gamma-like distributions. We derive analytical approximations for the gamma distribution parameters as a function of key physical processes on both wet and dry regimes in section 5. Section 5 also exemplifies how the shape of the daily precipitation distribution respond to changes of wet and dry regime dynamics. Section 6 explains how distributions change as a function of averaging interval, both subdaily and longer-than-daily precipitation averages. Finally, we conclude and discuss results on section 7 , with particular emphasis on global warming implications.

\section{Accumulation and daily precipitation distributions}

The main goal of this paper is to provide an explanation of why precipitation distributions can be well fitted by gamma-like distributions. We note that there are several other distributions that are also used to describe precipitation (e.g., Woolhiser and Roldán 1982; Cho et al. 2004; Papalexiou and Koutsoyiannis 2013; O'Gorman 2014; Kirchmeier-Young et al. 2016). It is not the intention of this study to distinguish the often subtle differences in fit among these distributions. For our purposes we employ the gamma distribution because parameters can be easily interpreted, provides a good enough fit in most cases, and we can track distribution changes quantitatively. In addition, as elaborated below, gamma distributions resemble accumulation distributions in mathematical form, so the similarities and differences between accumulation and daily precipitation distributions can be made more quantitative. To highlight parallels between daily precipitation (or other averaging intervals) with accumulations measured in millimeters, in the rest of the paper we look at the distribution of daily precipitation totals measured in millimeters, but a conversion to daily precipitation intensities measured in millimeters per day is straightforward.

While for longer averages the usual gamma distribution representation given in (1) is more instructive, for daily precipitation $P$, we prefer a representation of the form

$$
p_{P}=A P^{-\tau_{P}} \exp \left(-\frac{P}{P_{L}}\right),
$$

with $A=\Gamma\left(1-\tau_{P}\right)^{-1} P_{L}^{\tau_{P}-1}$ and $\Gamma$ the gamma function, because it highlights the parallels between daily precipitation and accumulation distributions. In this case $\tau_{P}\left[\tau_{P}=1-k\right.$ in (1), $\left.\tau_{P}<1\right]$ can be regarded as a power-law exponent governing the rate of decay of the distribution or probability density function (PDF) in the power-law range, and $P_{L}\left[P_{L}=\theta\right.$ in (1)] can be regarded as a daily precipitation cutoff scale, where the probability drops sharply.

Accumulations over precipitation events-from when precipitation exceeds a small threshold to when it drops below that threshold-are defined as

$$
s=\int_{0}^{t} R\left(t^{\prime}\right) d t^{\prime} .
$$

Here $t$ is the event duration, and $R\left(t^{\prime}\right)$ is the precipitation rate at time $t^{\prime}$ after the precipitating event has started. Accumulation distributions are well fitted in observations (Peters et al. 2010; Deluca and Corral 2014; MN18) and models (SN14; N17) by an expression of the form

$$
p_{s}=B s^{-\tau} \exp \left(-\frac{s}{s_{L}}\right),
$$

where $\tau$ is a power-law exponent (usually $>1$ ) governing the rate of decay of $p_{s}$ in its power-law range, $s_{L}$ is a cutoff scale for which the probability of extreme accumulation events decay sharply, and $B$ is a normalization factor. An expression resembling (4) has been derived analytically under simplifying assumptions by SN14, and will be further discussed in section 3a. We note that after (2) has been rearranged, daily precipitation and accumulation distributions formulas look similar, with the main difference being the sharper power-law exponent for accumulations $\left(\tau>1\right.$ and $\left.\tau_{P}<1\right)$. In addition, both (2) and (4) highlights the importance of the cutoffs $P_{L}$ and $s_{L}$ in controlling the probability of extremes. For $\tau>1$ there must a change in form for very small values of $s$, as discussed below, but this is smaller than can typically be observed. 
There are different approaches used in the literature to fit distributions to data. To fit accumulations and daily precipitation distributions we use a simple linear regression technique (see appendix A), with the estimated parameters being correlated with estimations using maximum likelihood (Thom 1958; Husak et al. 2007), and the method of moments (Fig. S1 in the online supplemental material). As notation, parameters estimated using the method of moments are denoted with a hat symbol (^), and unadorned parameters are estimated using the regression technique.

Figure 2a illustrates the key features of accumulation and daily precipitation distributions in observations. Here, accumulation and daily precipitation values are calculated using $1 \mathrm{~min}$ precipitation values from a DOE ARM site station located in Manus Island in the western tropical Pacific (Gaustad and Riihimaki 1996; Holdridge and Kyrouac 1997). A gamma distribution fit, (2), is overlaid on the daily precipitation distribution, and a fit given by (4) is overlaid on the accumulation distribution. The difference in the power-law exponent is immediately apparent, with accumulations decaying faster in the power-law range. The effect of the cutoff in limiting the probability of the largest events is also clear, especially compared to the respective dashed lines, which shows what the probability would be without the cutoffs. These main features are not restricted to this particular dataset. Similar features can be seen across a range of meteorological regimes for accumulations in (Peters et al. 2010; Deluca and Corral 2014), and for accumulation and daily intensity comparison in MN18.

\section{Modeling accumulation and daily precipitation distributions}

\section{a. Model setup}

To explain leading-order effects controlling daily precipitation distributions, we use a simple column model of the dry (nonprecipitating) and wet (precipitating) regimes based on a simplification of the column water vapor equation. This model, introduced by SN14, has been used to derive analytically the main aspects of accumulation distributions as a function of the relevant physics of the system (SN14; N17). In the model, precipitation is assumed to begin when column water vapor $q$ reaches a certain threshold $q_{c}$, as occurs in observations (Peters and Neelin 2006; Neelin et al. 2009; Ahmed and Schumacher 2015; Schiro et al. 2016; Kuo et al. 2018) and general circulation models (Sahany et al. 2012, 2014). Similarly, precipitation termination occurs when $q$ has decreased below another threshold $q_{\mathrm{np}}=q_{c}-b$, with $b$ a hysteresis parameter. The model is given by

$$
\begin{aligned}
& \frac{d q}{d t}=E+\bar{C}+D_{E} \eta \quad \text { (dry regime) } \\
& \frac{d q}{d t}=E+\bar{C}-R(q)+D_{P} \eta \quad \text { (wet regime) }
\end{aligned}
$$

Here, $E$ is a positive mean evaporation source, $\bar{C}$ is the climatological mean moisture convergence $(\bar{C}>0$ for convergence on the column), and $R(q)$ is the precipitation rate. Fluctuations in moisture convergence are parameterized by $D_{E} \eta$ in the dry regime, and $D_{P} \eta$ in the wet regime, with $\eta$ being Gaussian white noise $[\langle\eta(t)\rangle=0$, $\left.\left\langle\eta(t) \eta\left(t^{\prime}\right)\right\rangle=\delta\left(t-t^{\prime}\right)\right]$, with $\langle\cdot\rangle$ denoting expectation value), and $D_{E}$ and $D_{P}$ the noise amplitude in the respective regimes. For analytical simplicity we set $E+\bar{C}=0$ in the wet regime in what follows, as these terms are typically an order of magnitude (or more) smaller than $R(q)$. We derive analytically the effect that this makesessentially a slight modification to the cutoff scale $s_{L}$ of order $\delta \ll 1$-and show numerically its consequences in section $\mathrm{S} 1$ in the supplemental material. Another simplification of the model is that the moisture equation is independent of the large-scale flow. This implies that aspects related to the spatial organization of precipitation are not explicitly treated here. However, key features of the observed PDF of spatial clusters (Quinn and Neelin 2017) can be captured in models related to the one used here (Hottovy and Stechmann 2015; Ahmed and Neelin 2019). Despite simplifications, the essential elements explaining accumulation distributionsthe interplay between moisture convergence fluctuations and moisture loss by precipitation-are retained in the model.

We consider two variants of the precipitation parameterization $R(q)$ in the above model, for brevity denoted "on-off precipitation" and "ramp precipitation" given by

$$
\begin{aligned}
& R(q)=R_{0} \quad(\text { on-off precipitation) } \\
& R(q)=\alpha\left(q-q_{\mathrm{np}}\right) \quad(\text { ramp precipitation })
\end{aligned}
$$

In the on-off precipitation case, the existence of a threshold for precipitation onset and termination generates a first-passage process problem with solution for the distribution of accumulations (Redner 2001; SN14; N17). This solution corresponds to an inverse Gaussian (Tweedie 1957; Folks and Chhikara 1978), and is given by

$$
p_{s}=B \exp \left(-\frac{\bar{s}^{2}}{s_{L} s}\right) \exp \left(-\frac{s}{s_{L}}\right) s^{-3 / 2},
$$



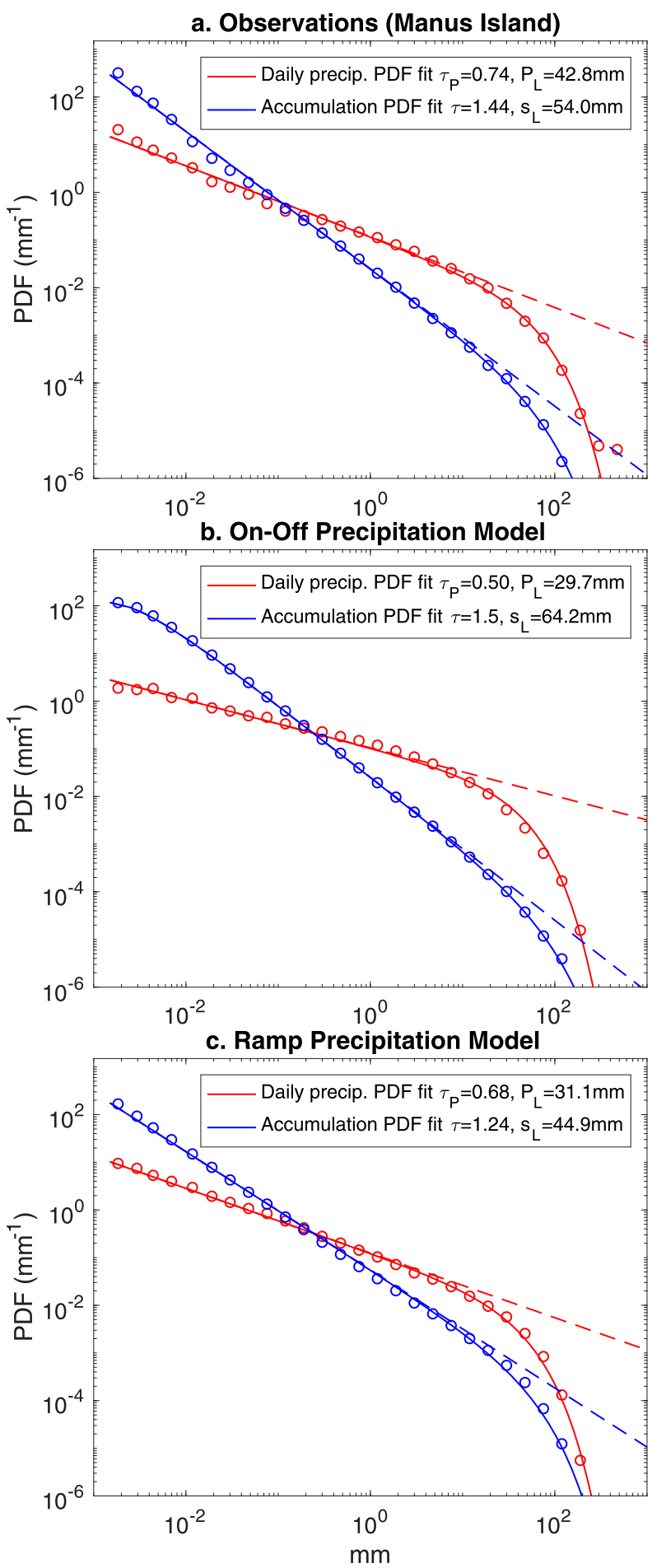

FIG. 2. Accumulations (blue circles) and daily precipitation (red circles) distributions in (a) observations at Manus Island $\left(2^{\circ} \mathrm{S}\right.$, $147^{\circ}$ E; January 1998 , September 2012), (b) generated by the model with on-off precipitation, and (c) generated by the model with ramp precipitation. Parameters of the models are $E=0.1 \mathrm{~mm} \mathrm{~h}^{-1}$, $\bar{C}=0.2 \mathrm{~mm} \mathrm{~h}^{-1}, b=0.2 \mathrm{~mm}, D_{E}=3 \mathrm{~mm} \mathrm{~h}^{-1 / 2}, q_{c}=65 \mathrm{~mm}$, with $R_{0}=9 \mathrm{~mm} \mathrm{~h}^{-1}$ and $D_{P}=17 \mathrm{~mm} \mathrm{~h}^{-1 / 2}$ in the model with on-off precipitation, and $\alpha=0.35 \mathrm{~h}^{-1}$ and $D_{P}=12 \mathrm{~mm} \mathrm{~h}^{-1 / 2}$ in the model with $B=\left(\bar{s} / \sqrt{\pi s_{L}}\right) \exp \left(2 \bar{s} / s_{L}\right)$. Note that (9) has the same shape as (4) for $s \gg \bar{s}^{2} / s_{L}$ (which is well satisfied except for small $s$ ), with $\tau=1.5$. Here $\bar{s}$ is the mean accumulation, and $s_{L}$ is the accumulation cutoff given by

$$
\bar{s}=b, \quad s_{L}=\frac{2 \sigma_{s}^{2}}{\bar{s}}=\frac{2 D_{P}^{2}}{R_{0}},
$$

with $\sigma_{s}^{2}$ the accumulation variance. Using (B2) we can show that

$$
s_{L} \propto \sqrt{t_{L}} D_{P}
$$

where $t_{L}$ is a similarly defined precipitating event duration cutoff (discussed below). From (11) we can see that $s_{L}$ (with same units as $q$ ) is proportional to the amplitude of moisture convergence fluctuations $\left(\propto D_{P}\right)$ in the wet regime. Under increasing moisture availability, these fluctuations are expected to scale with moisture (although locally dynamical effects can be important), which implies an extension of the power-law range and a large increase in probabilities for extremes under a global warming scenario (N17; Norris et al. 2019a). The ramp precipitation case provides a more realistic scenario for the precipitating regime, where precipitation acts as a negative feedback opposing further moisture increases. While an analytical solution for accumulation distributions in this case is not available, section S2 shows that (11) also holds numerically.

The physical mechanisms and mathematical derivation of why the accumulation distribution in the on-off precipitation case is given by (9) are discussed by SN14 and N17, and why more generally (4) should hold as a good approximation for observed accumulation distributions is discussed by N17 (see also N17 Fig. 4). For the reader's convenience we repeat the derivation of (9) in section S3 and summarize its main points here. In the onoff case the relation between column water vapor $q$ and accumulation $s$ can be made clearer by rewriting (6) as $d q=-d s+D_{s} \eta_{s} d s$, with $D_{s}=D_{P} / R_{0}^{1 / 2}$ and $\eta_{s}$ white

with ramp precipitation. Parameters are selected to generate similar accumulation and duration moment ratios $\left(\left\langle s^{2}\right\rangle /\langle s\rangle\right.$ and $\left\langle t^{2}\right\rangle /\langle t\rangle$, respectively, with $\langle\cdot\rangle$ denoting the expectation value) compared to Manus Island observations. All model parameters are also listed in Table S1. Accumulation and daily precipitation distributions are fitted following appendix A (blue and red solid lines, respectively) only taken into account bins with 10 or more counts, except for accumulations in the on-off precipitation case, where the analytical formula (9) is used. Blue and red dashed lines show only the powerlaw part of the fits to the accumulation and daily precipitation distributions. 
noise in the $s$ coordinate. This captures the main physical mechanisms governing the column water vapor equation within a precipitating event-the moisture converged to/diverged from the column $\left(D_{s} \eta_{s} d s\right)$ and the loss of moisture by precipitation accumulation $(-d s)$. If precipitation were not a moisture sink, there would be many trajectories where $q(s)$ lingers above the critical threshold for event termination, yielding a long tail for the probability of event accumulation $p_{s} \propto s^{-1.5}$ in this case. In reality, moisture loss by precipitation limits the long incursions of $q(s)$ above the threshold for event termination, as captured by the exponential term in (9) $\exp \left[-\left(s / s_{L}\right)\right]$. While there is no analytical solution for $p_{s}$ available in the ramp precipitation case, the two competing processes (fluctuations in moisture convergence and moisture loss due to precipitation) also occur, which yield a similar form for the accumulation distribution numerically, although with a modified power-law exponent.

By similar means as before, an analytical solution for the distribution of wet-spell durations can be calculated for the model with on-off precipitation (see appendix B). This solution also contains a cutoff for long durations $t_{L}$, which is relevant to certain approximations for understanding time-averaged intensities, discussed in section 4. Similarly, the dry-spell duration distribution also has an analytical solution (appendix B), although it should be noted that this solution is unrealistic for low $q$ values. In integrating the model (5) we simply set a rigid boundary at $q=1 \mathrm{~mm}$, with $q$ restored to the value at the previous time step if the boundary is reached. A more realistic treatment of the dry regime at low moisture values is implemented in a forthcoming paper. Nevertheless, the main way in which the dry regime affects daily precipitation distribution, namely in the different daily number of events distributions (section $4 \mathrm{~b}$ ) for mean moisture convergent $(\bar{C}>0)$ and divergent $(\bar{C}<0)$ conditions, is still captured by this setup.

\section{b. Daily precipitation and accumulation distributions in the model}

As an example of the accumulations and daily precipitation distributions arising from the setup in (5) and (6), we integrate the model, using both on-off and ramp precipitation variants, for 100 years, using the EulerMaruyama stochastic integration scheme (Gardiner 2009; Ewald and Penland 2009), with a time step of $0.6 \mathrm{~s}$. Parameters in the wet regime (see caption) are chosen to generate similar accumulation and duration moment ratios (see section S4) compared to observations. Parameters in the dry regime are similar to the ones chosen by SN14 and Abbott et al. (2016), with the value of $\bar{C}$ in line with observational estimates (Seager and Henderson 2013). The short time step of $0.6 \mathrm{~s}$ is needed to generate the smallest accumulation and daily precipitation values in the on-off precipitation case, such as to compare with Manus Island observations. This time step will be increased in subsequent sections. At each time step $q$ in (5) and (6) is calculated, from which it is decided whether it is precipitating $\left(q>q_{c}\right)$ or not $\left(q<q_{\mathrm{np}}\right)$, and from which a precipitation rate can be calculated using (7) and (8) as appropriate. Then accumulations and daily precipitation values can be calculated from the generated precipitation-rate time series.

Figures $2 \mathrm{~b}$ and $2 \mathrm{c}$ show the resulting accumulation and daily precipitation distributions, in the on-off precipitation case and the ramp precipitation case, respectively. Gamma distribution fits are overlaid on the simulated daily precipitation distributions. With some small differences, the resulting distributions show features much like what is seen in observations in both cases (Fig. 2a). Specifically, both daily precipitation and accumulation distributions have a cutoff scale, evident when comparing to the dashed lines indicating no cutoff, and the power-law range in the daily precipitation distributions is less steep than for accumulations. Given the relative simplicity of the setup, these results are encouraging and suggest that the physics included in the models is adequate to explain fundamental processes underlying these distributions. For the sake of obtaining analytical expressions, we use the on-off precipitation parameterization in most of what follows. The ramp precipitation model produces results that are qualitatively similar to the on-off precipitation model (see supplementary information).

\section{What explains the shape of the daily precipitation distribution}

In this section we provide a rationale for how daily precipitation distributions get their shape. The two ingredients are (i) the distribution of accumulations and (ii) the daily number of events distribution. Point (i) depends entirely on wet regime properties, while point (ii) depends on both wet and dry regime dynamics. This allows a conceptual separation of mechanisms between wet and dry regime processes controlling daily precipitation distributions. We focus on daily precipitation here, with section 6 showing other averaging intervals.

The main requirement for this partition to work is that $t_{\text {avg }} \gg t_{L}$, with $t_{\text {avg }}$ the averaging interval (1 day in this case), and $t_{L}$ the wet-spell duration cutoff $\left[t_{L}=2 D_{P}^{2} / R_{0}^{2}\right.$ in the on-off precipitation case; (B2)]. This holds 
reasonably well in most regions, although it may become more difficult to meet for regions or seasons with a preponderance of stratiform precipitation (small $R_{0}$ ) or large moisture convergence fluctuations in the wet regime (large $D_{P}$ ). This requirement is put in place so the whole accumulation distribution can be sampled during $t_{\mathrm{avg}}$, and that boundary effects from when a day starts and ends are small. This requirement also implies that the number of events and accumulation distributions are asymptotically independent. In this section and in the derivation of analytical results in section 5 we assume that we are in a regime where $t_{\text {avg }} \gg t_{L}$ holds perfectly. We test deviations from this requirement for $t_{\mathrm{avg}}=1$ day numerically in section 5. In practice, subdaily precipitation distributions are the most affected. However, some insight can still be gained for these distributions, as discussed in section $6 \mathrm{a}$.

\section{a. Distribution of daily precipitation from the accumulation distribution}

The probability distribution of accumulations, (9), provides key information about the distribution of daily precipitation (and other averaging intervals). The sum over accumulations in a given day will yield the precipitation over that day, so it is useful to sort by the days that contain a given number of events $n$. Denoting $P_{n}$ as the total precipitation in days with $n$ events, then

$$
P_{n}=\sum_{i}^{n} s_{i}
$$

This is a quantity with clear properties that provides a step on the way to obtaining insight into the daily precipitation distribution. In calculating it from data, one simply sorts by the number of times it rained during a day. The distribution of $P_{n}$ values, here denoted as the "conditional daily precipitation distribution" $p_{n}$ is given by

$$
p_{n}=B_{n} \exp \left(-\frac{n^{2} \bar{s}^{2}}{s_{L} P_{n}}\right) \exp \left(-\frac{P_{n}}{s_{L}}\right) P_{n}^{-3 / 2},
$$

with $B_{n}=\left(n \bar{s} / \sqrt{\pi s_{L}}\right) \exp \left(2 n \bar{s} / s_{L}\right)$. This formula for $p_{n}$, (13), is obtained from knowledge of the accumulation distribution, (9). See appendix $\mathrm{C}$ for details of this derivation. This distribution $p_{n}$ has the same shape as the accumulation distribution $p_{s}$ in (9), but with mean $\bar{P}_{n}=n \bar{s}$. Similar to the accumulation distribution, $p_{n}$ only depends on processes occurring while precipitating. Figure 3 a shows examples of $p_{n}$ distributions for different $n$ values. Since it typically rains a larger daily precipitation amount in days with many accumulation events,
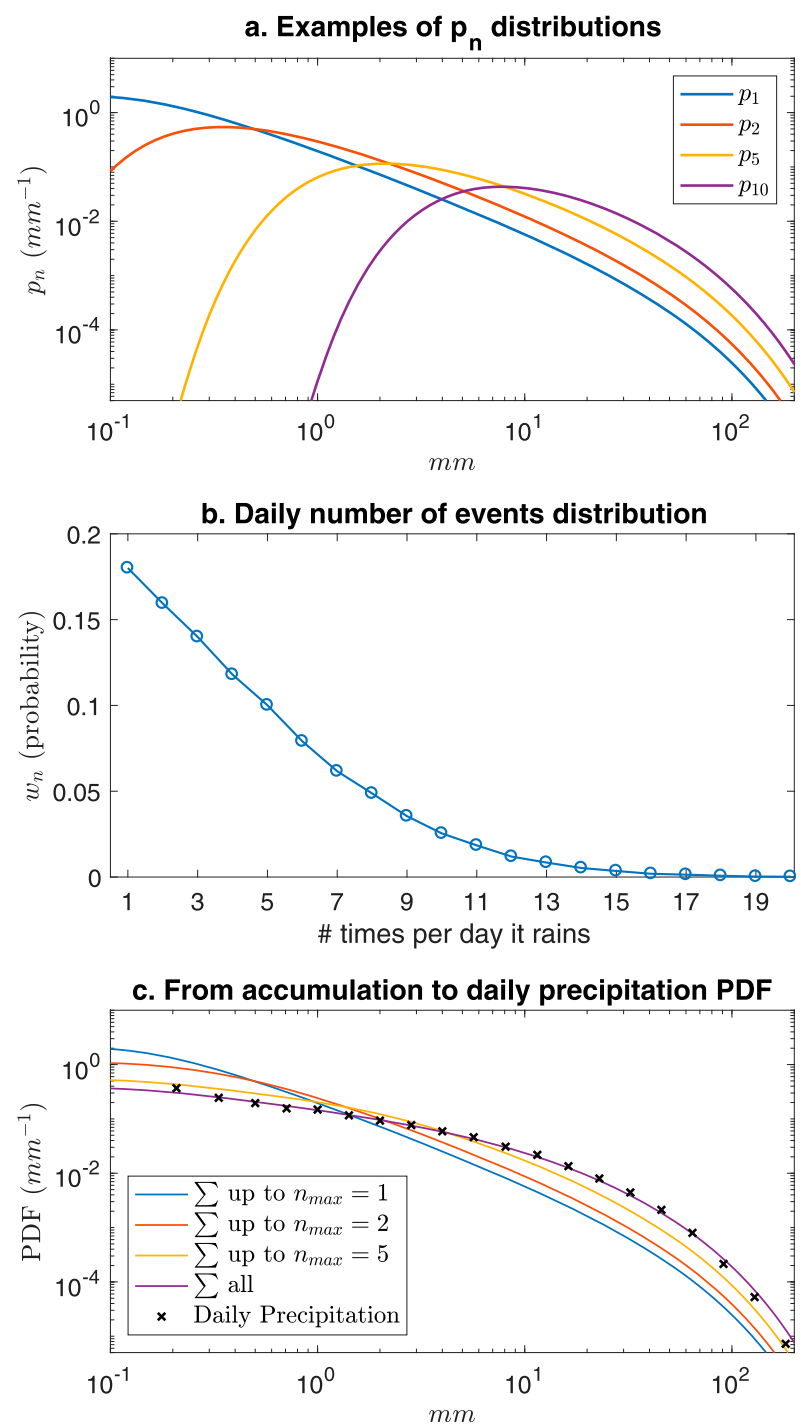

FIG. 3. (a) Examples of $p_{n}$ distributions for different values of $n$, for parameters $s_{L}=45 \mathrm{~mm}$ and $\bar{s}$ equal to the mean of the accumulations in the integration used in (b). (b) Daily number of events distribution calculated from a 500-yr run of the model, (5)-(7), with parameters $E=0.1 \mathrm{~mm} \mathrm{~h}^{-1}, \bar{C}=0, D_{P}=$ $15 \mathrm{~mm} \mathrm{~h}^{-1 / 2}, D_{E}=3 \mathrm{~mm} \mathrm{~h}^{-1 / 2}, R_{0}=9 \mathrm{~mm} \mathrm{~h}^{-1}$ and $b=1 \mathrm{~mm}$ [note that $s_{L}=\left(2 D_{P}^{2} / R_{0}\right)=45 \mathrm{~mm}$ ]. All model parameters are also listed in Table S1. (c) Daily precipitation distribution calculated from the same model run, and increasingly better approximations of (14), $\sum_{n}^{n_{\max }} w_{n} p_{n}$. The daily precipitation calculated directly from the integration is denoted by black $\times$ symbols.

the $p_{n}$ distributions, (13), show lower probability for small daily precipitation amounts and higher probability for large daily precipitation amounts for increasing $n$. Consequently, as $n$ gets large the resulting $p_{n}$ distributions look less asymmetric, that is, evolve to be less skewed (skewness decreases with $n$ as $n^{-1 / 2}$ ), with mean increasing proportionally to $n$, and with fixed $s_{L}$, although it should be noted that the interpretation 
of $s_{L}$ as a distinct cutoff in $P_{n}$ is clear only for low enough $n$.

\section{b. Daily number of events distribution}

From the $p_{n}$ distribution, the daily precipitation distribution can be calculated as the mixture

$$
p_{P}=\sum_{n}^{n_{\max }} w_{n} p_{n}
$$

with $w_{n}$ (the weights of the mixture; $\sum_{n=1}^{n_{\max }} w_{n}=1$ ) the daily number of events distribution. See FrühwirthSchnatter (2006) for more details on mixture distributions. Figure $3 b$ shows the daily number of events distribution calculated from a long 500-yr integration of (5) and (6). This distribution gives the information on the fraction of wet days with $n$ events. For this particular choice of parameters (see caption) it rains most often once per day ( $\sim 18 \%$ of rainy days), and the probability decreases monotonically for larger $n$. This quantity depends on both wet and dry regimes as shown in section 5b. An analytical solution for this distribution is not presently available, although it can be readily calculated from observations or from model integrations.

\section{c. Resulting daily precipitation distribution}

Figure $3 \mathrm{c}$ shows graphically how daily precipitation distributions get their shape as we increase $n_{\max }$ in (14). Only considering $n=1$ the resulting distribution is equal to the accumulation distribution. As we increase $n_{\max }$ we observe that small size values lose probability, and large daily precipitation values increase in probability, as less asymmetric $p_{n}$ distributions (Fig. 3a) are incorporated. This process flattens out the resulting distribution, generally resulting in a daily precipitation power-law exponent $\tau_{P}$ that is smaller than the accumulation distribution power-law exponent. This implies that the resulting daily precipitation distribution can often be fitted by gamma distributions $\left(\tau_{P}<1\right)$. We note that the apparent power-law range in the gamma distribution is not precisely a power law as in the accumulation solution. The power-law approximation holds well because a true scale free range is being modified by a procedure that does not introduce any dominant scale, thus leaving a range that remains essentially scale free. As all $w_{n} p_{n}$ are included, the distribution resulting from (14) is very similar to the one calculated directly from the integration. This leads to a dependence of the daily precipitation distribution on the parameters of the underlying accumulation distributions, as further elaborated below. This simple model thus provides a rationale for how daily precipitation PDFs arise, and why they can be fitted by gamma distributions.

\section{Analytical approximation of daily precipitation distribution parameters}

\section{a. Analytical approximations}

In this section we provide analytic expressions for the mean and variance of daily precipitation distributions, and use these expressions to estimate the gamma distribution parameters as a function of the underlying accumulations and daily number of events distributions. The daily precipitation mean $\bar{P}$ and variance $\sigma_{P}^{2}$ are given by (see appendix D for derivation)

$$
\begin{aligned}
\bar{P} & =\bar{w} \bar{s}, \\
\sigma_{P}^{2} & =\sigma_{w}^{2} \bar{s}^{2}+\bar{w} \sigma_{s}^{2},
\end{aligned}
$$

where $\bar{s}$ is the mean storm accumulation, $\sigma_{s}^{2}$ is the accumulation variance, and $\bar{w}$ and $\sigma_{w}^{2}$ are the mean and variance of the number of precipitating events in a day (the first two moments of the daily number of events distribution in section $4 \mathrm{~b}$ ), considering only wet days. This shows that if the mean number of precipitating events occurring per day increases and/or the mean accumulation per event increases, then the mean daily precipitation increases. Similarly, the daily precipitation variance has weighted contributions from both the accumulation variance and the variance of the number of precipitating events per day.

From (15) and (16), expressions for the daily precipitation power-law exponent $\tau_{P}$ and cutoffs $P_{L}$ can be obtained using the method of moments (see appendix D) as

$$
\begin{aligned}
& \hat{P}_{L}=\frac{\sigma_{w}^{2}}{\bar{w}} \bar{s}+\frac{s_{L}}{2}, \\
& \hat{\tau}_{P}=1-\frac{\bar{w}}{\frac{\sigma_{w}^{2}}{\bar{w}}+\frac{s_{L}}{2 \bar{s}}} .
\end{aligned}
$$

We should note that maximum likelihood estimates of $\tau_{P}$ and $P_{L}$ will generally differ from the estimates calculated above, but they will be proportional (see Fig. S1). As previously stated, $\bar{w}$ and $\sigma_{w}^{2}$ depend on both dry and wet regimes, and encapsulate the effects arising from aggregating different accumulation events in the averaging interval $t_{\text {avg }}$ of interest. On the other hand, $s_{L}$ and $\bar{s}$ depend only on the wet regime, and their contribution to $\hat{P}_{L}$ and $\hat{\tau}_{P}$ is independent from $t_{\text {avg. }}$. That is, $\bar{s}$ and $s_{L}$ contribute the same to daily or monthly precipitation statistics, with the difference between statistics for different averaging intervals being accounted by $\bar{w}$ and $\sigma_{w}^{2}$.

\section{b. Exploring the parameter space}

In this section we investigate the influence of both wet and dry regimes in the daily precipitation distribution 
a. Wet regime effects: $\hat{P}_{L}$ vs $s_{L}$

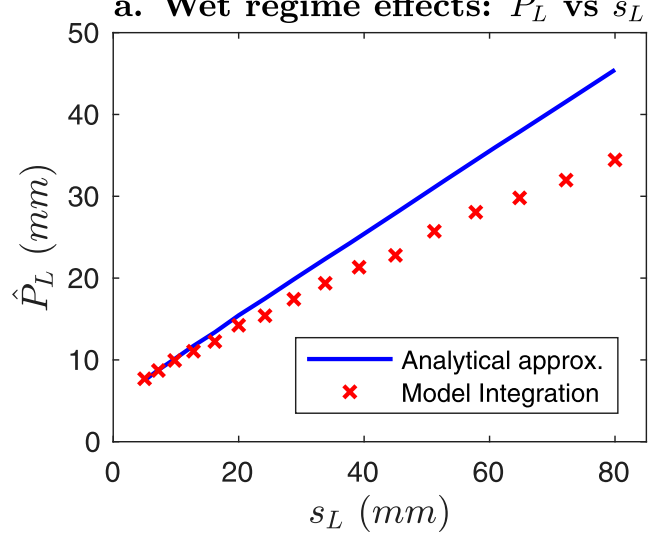

c. Wet regime effects: $\hat{\tau}_{P}$ vs $s_{L}$

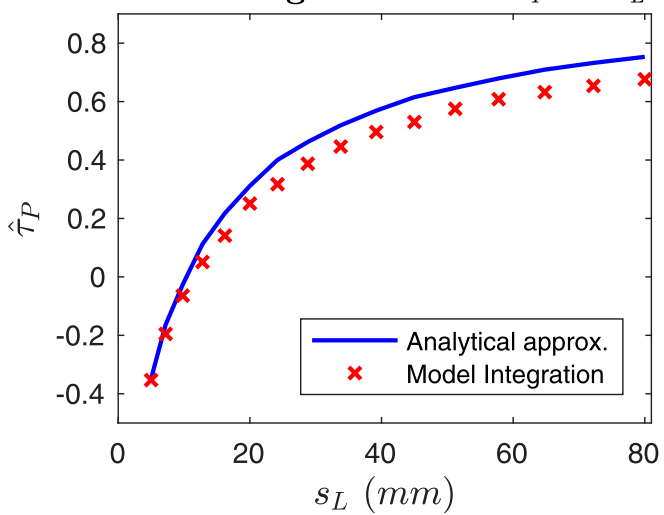

b. Dry regime effects: $\hat{P}_{L}$ vs $\bar{C}$

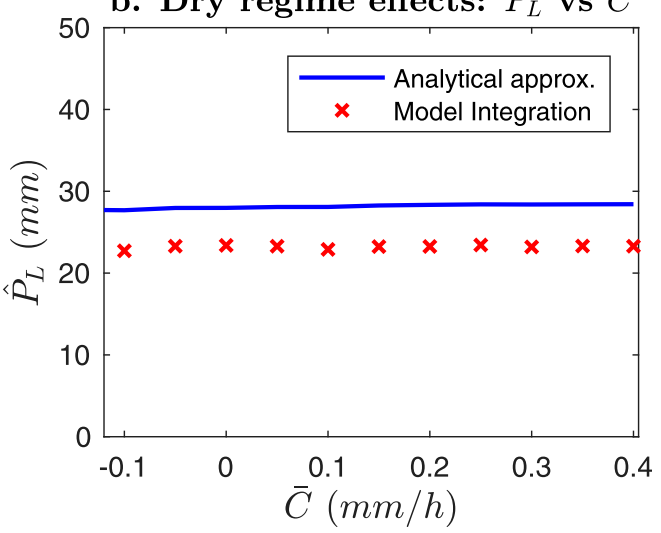

d. Dry regime effects: $\hat{\tau}_{P}$ vs $\bar{C}$

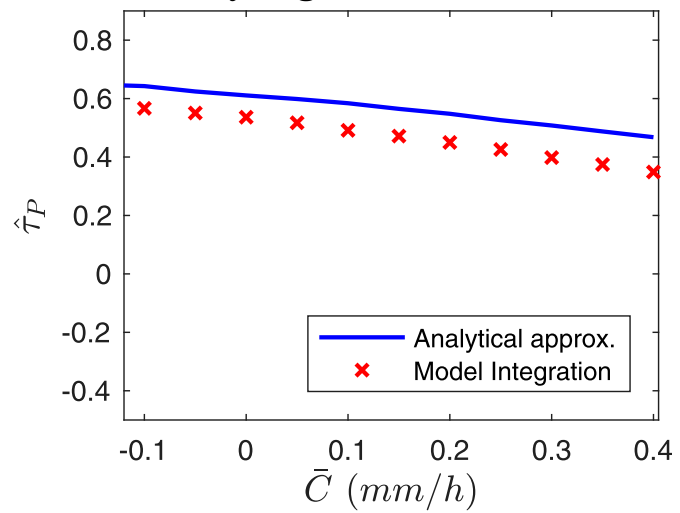

FIG. 4. Daily precipitation cutoff scale $\hat{P}_{L}$ as a function of parameters associated with (a) the wet and (b) the dry regimes. (c),(d) As in (a) and (b), but for the exponent $\hat{\tau}_{P}$. For the wet regime cases in (a) and (c), $\hat{P}_{L}$ and $\hat{\tau}_{P}$ values are calculated from different 500 -yr model integrations with the on-off precipitation parameterization for different values of $s_{L}=2 D_{P}^{2} / R_{0}\left(R_{0}\right.$ fixed at $\left.10 \mathrm{~mm} \mathrm{~h}^{-1}\right)$, and the dry regime fixed with parameters $E=0.1 \mathrm{~mm} \mathrm{~h}^{-1}, \bar{C}=0$, and $D_{E}=3 \mathrm{~mm} \mathrm{~h}^{-1 / 2}$. For the dry regime cases in (b) and (d), $\hat{P}_{L}$ and $\hat{\tau}_{P}$ values are calculated from 500-yr model integrations with on-off precipitation for different values of $\bar{C}$, with remaining values kept fixed at $E=0.1 \mathrm{~mm} \mathrm{~h}^{-1}$, $D_{E}=3 \mathrm{~mm} \mathrm{~h}^{-1 / 2}, R_{0}=10 \mathrm{~mm} \mathrm{~h}^{-1}$, and $D_{P}=12 \mathrm{~mm} \mathrm{~h}^{-1 / 2}$. In all cases $q_{c}=65 \mathrm{~mm}$ and $b=1 \mathrm{~mm}$. All model parameters are also listed in Table S1. Analytical approximations to these values (section 5a) are plotted as blue lines.

power-law exponent and cutoff. In each case we use a number of 500-yr integrations of (5) and (6) for different parameters in the dry and wet regimes, using an integration time step of one minute for speed. Analytical formulas (17) and (18) are used to interpret the results.

\section{1) DEPENDENCE ON WET REGIME}

Keeping the dry regime fixed, Figs. 4a,c show the dependence of the resulting daily precipitation gamma distribution parameters on different $s_{L}$ values. As shown in (11), $s_{L}$ is proportional to the amplitude of moisture converge fluctuations $\left(\propto D_{P}\right)$ in the wet regime. Figure 4a shows that the accumulation and daily precipitation cutoffs are very well correlated. That is, increases in the accumulation cutoff are associated with increases in the daily precipitation cutoff. This relation between cutoffs is also reproduced in the ramp precipitation case (Fig. S4a). This dependence can be explained from (17) by noting that the expression $\sigma_{w}^{2} / \bar{w}$ is of order 1 for daily time scales (Fig. S5), and that typically $s_{L} \gg \bar{s}$. This implies that for daily time scales $P_{L}$ is set by the wet regime with

$$
\hat{P}_{L} \propto s_{L}
$$

This provides an explanation for the correlation between accumulation cutoff and daily precipitation cutoff values observed in weather station data in the United States (MN18). While the influence of the first term in the righthand side of the $\hat{P}_{L}$ equation, (17) is small at daily time scales, it will increase in importance as we increase the averaging interval. This is discussed in section 6 . 
Unlike the accumulation power-law exponent $\tau$, which always has a value of 1.5 in the on-off precipitation case, the daily precipitation power-law exponent $\tau_{P}$ exhibits an important dependence on model parameters. Figure $4 \mathrm{c}$ shows that $\hat{\tau}_{P}$ is larger for increases in moisture convergence fluctuations amplitude (larger $s_{L}$ ) all else being equal. Similar behavior is found in the ramp model (Fig. S4c), although the range of variation of $\hat{\tau}_{P}$ is more constrained in that case.

The left column of Fig. 5 shows the distributions associated with the left column of Fig. 4 for values $D_{P}=$ $10,15,20 \mathrm{~mm} \mathrm{~h}^{-1 / 2}$ ( $R_{0}$ fixed), corresponding to $s_{L}=20$, $45,80 \mathrm{~mm}$, respectively, with dry regime parameters fixed $(\bar{C}=0)$. Figure 5 a shows the accumulation distributions, which are clearly different due to the different cutoff. Changes in wet regime dynamics also impact the daily number of events distribution. Larger moisture convergence fluctuations in the wet regime (larger $D_{P}$ ) are associated with fewer total events (Fig. S6a), and to a number of events distribution more weighted toward fewer events per day (Fig. 5c). This apparently occurs because for increasing $D_{P}$ the system spends more time precipitating, which results in larger but fewer events. This implies a larger contribution from more asymmetric conditional daily precipitation distributions $p_{n}(13)$ in (14), which results in a steeper daily precipitation power-law range for larger $D_{P}$ (or $s_{L}$ ) (Fig. 5e), which agrees with numerical and analytical results (Fig. 4c). It can also be seen that increases in $s_{L}$ translates to similar increases in the daily precipitation cutoff (Fig. 5e), in agreement with (19).

\section{2) DEPENDENCE ON DRY REGIME}

In this section we explore the dependence of the gamma distribution parameters for variations in the dry regime. Keeping the wet regime fixed (to $s_{L}=45 \mathrm{~mm}$ ), Figs. $4 \mathrm{~b}$ and $4 \mathrm{~d}$ show the dependence of $\hat{\tau}_{P}$ and $\hat{P}_{L}$ on climatological column moisture convergence $\bar{C}$. This variable is the main control on how frequently it precipitates (Fig. S6b), with $\bar{C}>0$ indicating precipitation prone regions, and $\bar{C}<0$ indicating dry regions (e.g., high pressure subtropical regions). While $\bar{C}$ controls how often it precipitates, it does not have a sizable effect on the value of the daily precipitation cutoff (Fig. 4b). The power-law exponent $\tau_{P}$ does have a slight dependence on $\bar{C}$, with a less steep power-law exponent in regions of positive climatological moisture convergence, all else fixed. The overall behavior is replicated in the ramp precipitation case (Figs. S4b,d).

The right panel in Fig. 5 shows the distributions associated with the right panel in Fig. 4 for $\bar{C}=$ $-0.1,0.1,0.3 \mathrm{~mm} \mathrm{~h}^{-1}\left(E+\bar{C}=0,0.2,0.4 \mathrm{~mm} \mathrm{~h}^{-1}\right)$, corresponding to mean moisture divergent, lightly and strongly convergent conditions, respectively. The wet regime is fixed $\left(s_{L}=45 \mathrm{~mm}\right)$. Since the wet regime is fixed, the three cases considered here have the same accumulation distribution (Fig. 5b). Consequently, the dry regime may only affect daily precipitation distributions through its effect on daily number of events distributions (Fig. 5d). As expected, the number of events distribution is weighted toward fewer events per day for mean moisture divergent conditions, as raining events are rarer in that case. This implies that for the $\bar{C}=-0.1 \mathrm{~mm} \mathrm{~h}^{-1}$ case there is a larger contribution of more asymmetric conditional daily precipitation distributions $p_{n},(13)$, that make up the daily precipitation distribution, (14). This results in a steeper power-law range in this case, in agreement with numerical and analytical results in Fig. 4d.

\section{3) SuMmary}

In summary, the wet regime controls the daily precipitation cutoff $P_{L}\left(P_{L} \propto s_{L}\right)$. Both wet and dry regimes have influence on the power-law exponent $\tau_{P}$-steeper power law for larger $D_{P}$ in the wet regime and/or decreasing $\bar{C}$ (i.e., mean moisture divergent conditions in the dry regime)-all else being equal. Wet regime parameters can cause more variation in $\tau_{P}$ (over the range considered), but the presence of the dry regime is essential to setting the difference between $\tau_{P}$ and the exponent for accumulations $\tau$.

\section{4) CAVEAT ON ANALYTICAL APPROXIMATIONS}

It should be noted that the analytical approximations (17) and (18) provide a good understanding of the numerical values shown in Fig. 4, but the comparison is not perfect. This occurs because as $s_{L}$ increases in Figs. $4 \mathrm{a}$ and $4 \mathrm{c}$ so does $t_{L}$, and the condition $t_{\mathrm{avg}} \gg t_{L}$ implies that (17) and (18) progressively becomes a worse approximation. For the parameters in Fig. 4, when $s_{L}$ is equal to $80 \mathrm{~mm}, t_{L}$ is equal to $8 \mathrm{~h}$, a value not that well separated from 1 day. The analytical approximations hold better as $t_{\text {avg }}$ increases as can be seen in Fig. S7. An implication of the $t_{\text {avg }} \gg t_{L}$ requirement is the asymptotic independence between the accumulation and number of events distributions. This is a simplification, as short events tend to preferentially occur in days with many events, and longer events to preferentially occur in days with few events. Despite this, leadingorder effects are well captured by (17) and (18), and can be used to provide insight into how gamma distribution parameters respond to dry and wet regime physics.

\section{How precipitation distributions change as a function of averaging interval}

As can be seen in (17) and (18), there are two main effects that explain precipitation distributions over a 
Wet regime effects

a. Accumulation PDFs

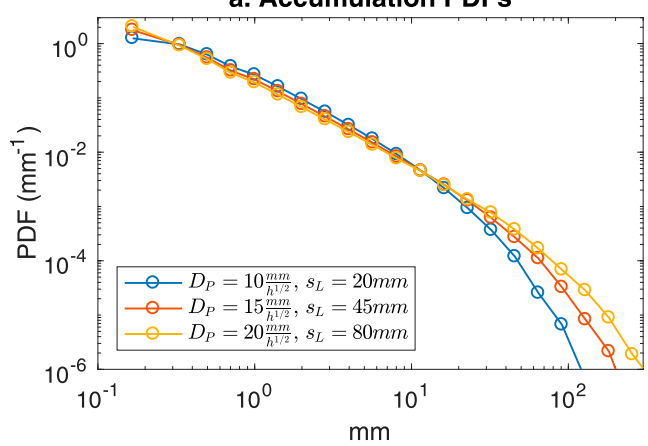

c. Daily number of events distribution

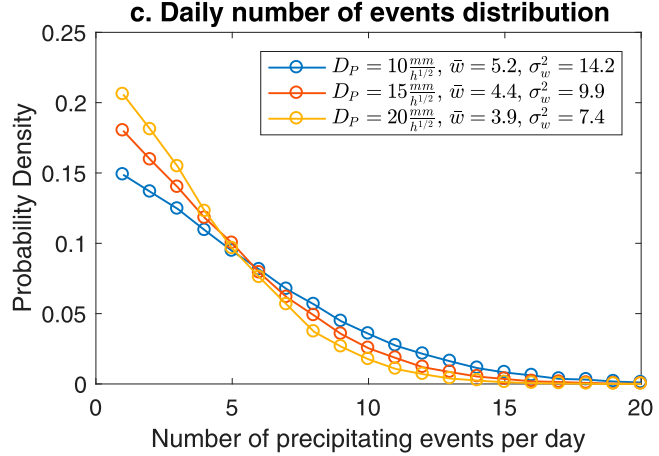

e. Daily Precipitation PDFs

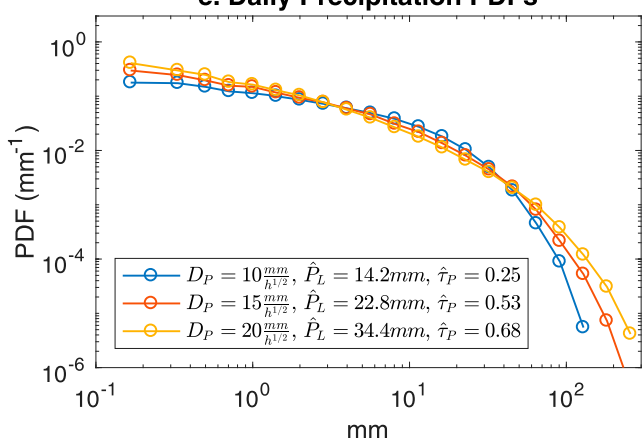

Dry regime effects

b. Accumulation PDFs

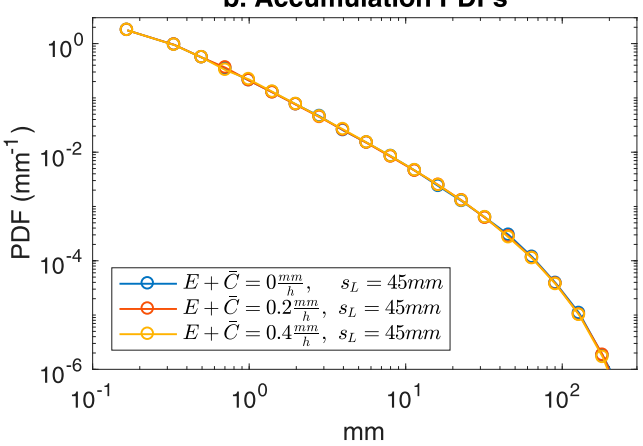

d. Daily number of events distribution

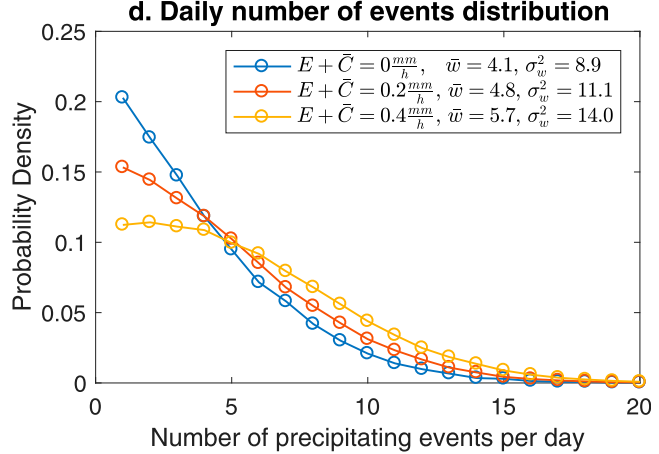

f. Daily Precipitation PDFs

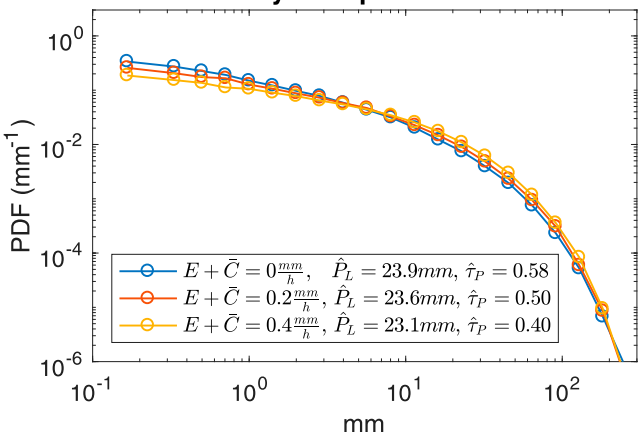

FIG. 5. (a) Accumulation distributions for three 500-yr runs of (5) and (6) with different amplitude of moisture convergence fluctuations in the wet regime $\left(D_{P}=10,15,20 \mathrm{~mm} \mathrm{~h}^{-1 / 2}\right)$ with the dry regime fixed. (b) Accumulation distributions for three 500-yr runs of (5) and (6) with different mean moisture convergence in the dry regime $\left(E+\bar{C}=0,0.2,0.4 \mathrm{~mm} \mathrm{~h}^{-1}\right)$ with the wet regime fixed. (c),(d) Daily number of events distribution calculated from the same run as (a) and (b). (e) Resulting daily precipitation distribution arising from the interplay between (a) and (c). (f) Resulting daily precipitation distribution arising from the interplay between (b) and (d). Common parameters used for both regimes are $R_{0}=10 \mathrm{~mm} \mathrm{~h}^{-1}, D_{E}=3 \mathrm{~mm} \mathrm{~h}^{-1 / 2}$, and $b=1 \mathrm{~mm}$. The dry regime is fixed with $\bar{C}=0$ in the left column, and the wet regime is fixed with $D_{P}=15 \mathrm{~mm} \mathrm{~h}^{-1 / 2}$ in the right column. All model parameters are also listed in Table S1.

fixed averaging interval. The first one, which may be thought as a fundamental effect arising from the lifetime of individual storms, is the distribution of accumulations, which impacts $\hat{\tau}_{P}$ and $\hat{P}_{L}$ mainly by the value of the underlying accumulation cutoff $s_{L}$. This contribution is completely independent from averaging considerations. The second effect, which may be thought as arising from the temporal aggregation of individual storms within the fixed averaging time of interest, will include the effects from averaging. Here those effects are encapsulated by the mean $\bar{w}$ and variance $\sigma_{w}^{2}$ of the number of precipitating events in a particular averaging interval. For fixed model parameters, this second effect is the one that controls the statistics as a function of averaging interval.

Before proceeding, we point out some practical consequences that the resolution of observational data has 
on observed distributions. In the model world it is possible to generate precipitation data at high temporal resolution. In contrast, observational data is often only available through already discretized accumulated values (e.g., $1 \mathrm{~h}$ precipitation). The model with on-off precipitation generates an accumulation power-law exponent $\tau=1.5$ and cutoff $s_{L}=2 D^{2} / R_{0}$ when precipitation is calculated using instantaneous values generated every $d t$ interval (with $d t$ small). Using coarser temporal resolution, as in observations, results in power-law exponents $<1.5$ (e.g., 1.3, when averaging from 1 to $15 \mathrm{~min}$; see Fig. S8) and also in somewhat smaller values of $s_{L}$. This effect occurs for reasons analogous to the changes in time-averaged intensities: coarse graining the data before computing event accumulations tends to cause some small events that, if observed at high resolution, are interrupted by short dry spells to instead be counted as larger events. This should be taken into account when evaluating distributions in observations.

\section{a. Subdaily precipitation distributions}

The study of subdaily precipitation statistics is an active area of research (e.g., Lenderink and van Meijgaard 2008; Westra et al. 2014; Barbero et al. 2017; Prein et al. 2017), so it seems useful to test to what extent the insight gained at daily time scales can be translated to subdaily scales as well. As previously stated, expressions (17) and (18) are approximations to gamma distribution parameters under the assumption that the averaging interval $t_{\text {avg }}$ is much longer than the local storm duration cutoff $t_{L}$; see (B2). This assumption becomes progressively worse as the averaging interval is decreased. This occurs because long events, that preferentially contribute to the tail of the accumulation distribution, cannot be fully sampled in a short averaging interval. Despite this, qualitative statements based on how precipitation distributions arise (see section 4) may still provide insight.

Figure 6a shows the accumulation, 3-h, 12-h, and daily precipitation distributions calculated from almost 15 years of 1 min data from Manus Island station. It can be seen that a power law with a cutoff is a good fit in all cases. All the time-averaged distributions have a gentler powerlaw range decay compared to accumulations, and the power-law exponent decreases as we increase the averaging interval. The cutoff scale (as would be expected) increases with averaging interval, although the increase is relatively slow.

Figure 6a may be compared to Fig. 6c generated by the model with on-off precipitation. We integrate the model for 100 years (for consistent statistics) using a time step of $0.6 \mathrm{~s}$ (necessary to resolve short time variations and small precipitation increments) from which 1-min totals are calculated, to make the output more consistent with observations in computing the distributions. The parameters (see caption) were chosen to give similar accumulation and duration moment ratios as observations $\left(\left\langle s^{2}\right\rangle /\langle s\rangle\right.$ and $\left\langle t^{2}\right\rangle /\langle t\rangle$, respectively), and a value of $\bar{C}=0.2 \mathrm{~mm} \mathrm{~h}^{-1}$ was used to be consistent with the mean moisture convergent conditions of Manus Island. Besides that, no other attempts to tune parameters of the model were made. While there are differences in the $\tau_{P}$ and $P_{L}$ parameters calculated, Fig. 6c shows good qualitative agreement with observations. Specifically, all the time-averaged distributions are well fitted by gamma distributions, with the correct ranking of power-law exponents simulated, and an (albeit faster) increase in the cutoff with averaging interval.

\section{b. Longer-than-daily precipitation distributions}

A comparison between observations and model for longer averages can also be made. In this case we use 64 years of hourly precipitation data from the Miami Airport station available from the NOAA/NCEI Climate Data Online system. The minimum instrumental resolution is $0.254 \mathrm{~mm} \mathrm{~h}^{-1}$ (compared to $0.1 \mathrm{~mm} \mathrm{~min}^{-1}$ in Manus Island) and observations are given in multiples of $0.254 \mathrm{~mm} \mathrm{~h}^{-1}$. Figure $6 \mathrm{~b}$ shows this station's accumulation, daily, 2-day, and 5-day precipitation distributions. Some of the same features as the subdaily precipitation distributions are seen. All time-averaged distributions have a smaller power-law exponent than accumulations, and the power-law exponents decrease in magnitude as the averaging interval is increased. In addition, the cutoffs increase slowly with averaging interval. Note that the accumulation distribution powerlaw exponent being smaller (in magnitude) than 1 most likely occurs due to the observations being given at $1 \mathrm{~h}$ intervals (see Fig. S8; also see section S4).

Noting that both Manus Island and Miami stations are located in regions with plentiful convection, it is worth asking whether accumulation and temporally averaged precipitation distributions behave similarly in regions and seasons dominated by frontal precipitation. We repeat the analysis leading to Fig. $6 \mathrm{~b}$ for two other stations, one located in the northeastern United States and the other in Southern California, for both annual and the extended winter (November-April) season. In all cases the main features seen in Fig. 6b-the general shape of the distributions, the sharper power-law exponent for accumulation compared to daily precipitation distributions, and the decrease of $\tau_{P}$ for increasing $t_{\text {avg }}$-are also present in the other locations and season analyzed (Fig. S9). This suggests the robustness of these results to geographical location and main type of precipitation.

As before, we integrate the model with on-off precipitation with parameters chosen such as to generate 
Sub-daily precipitation distributions
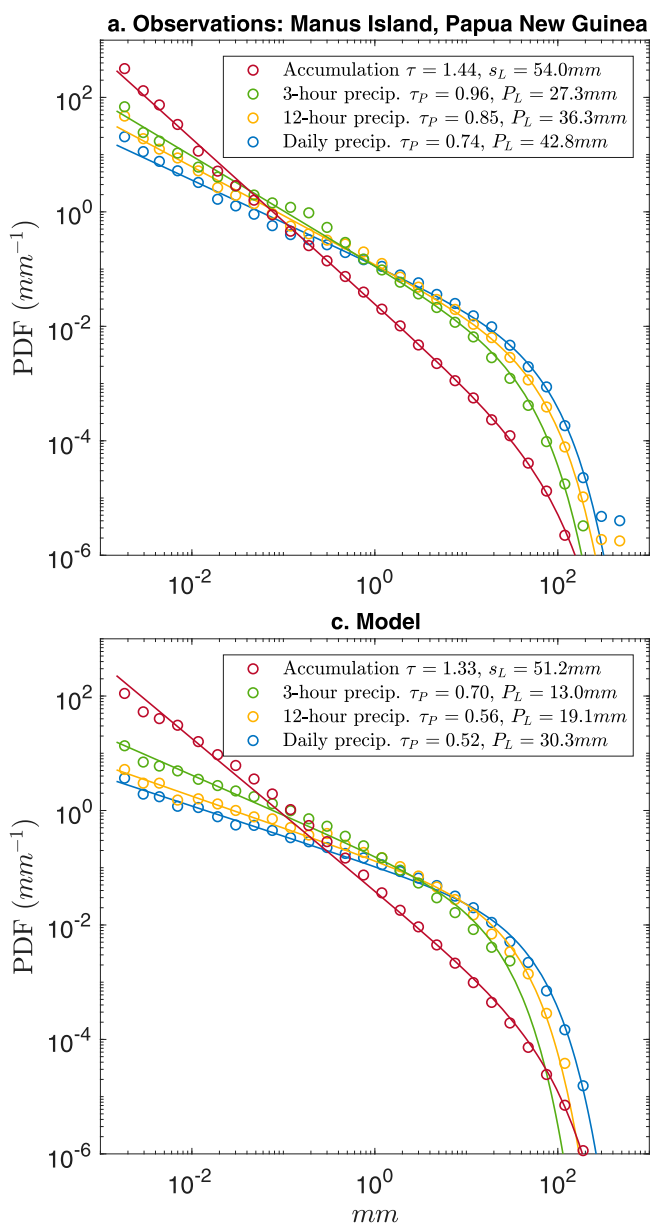

Longer than daily precipitation distributions
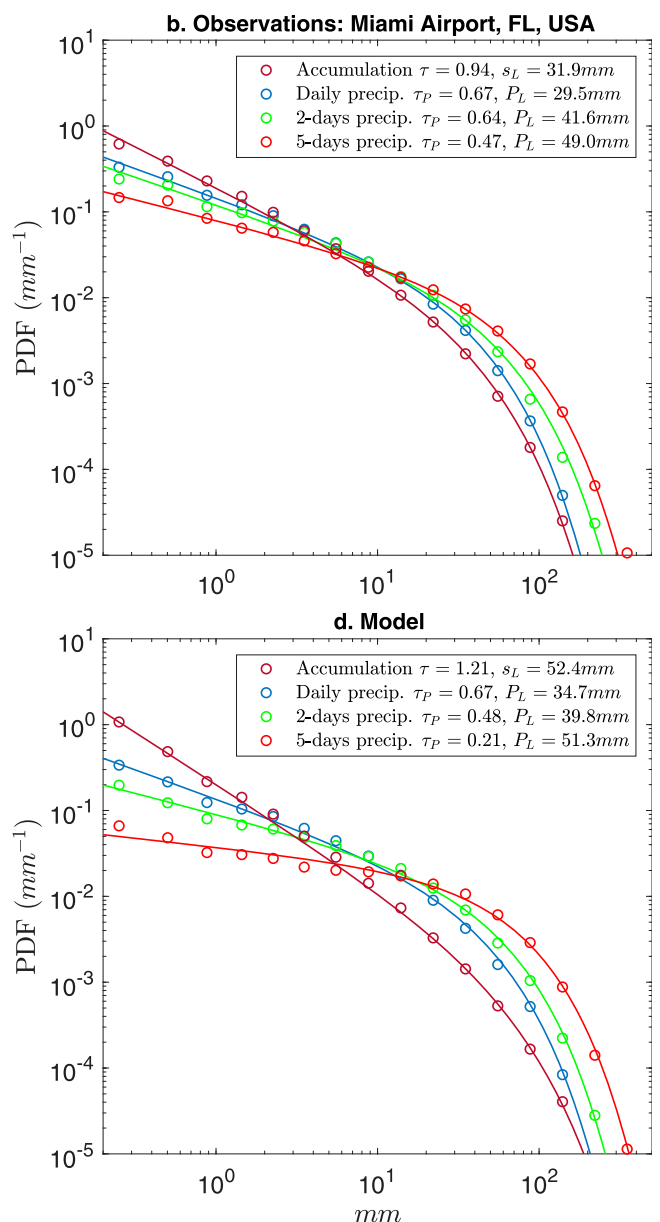

FIG. 6. (a) Accumulation, 3-h, 12-h, and daily precipitation distributions calculated using 1 min data (1 Jan 1998 to 14 Sep 2012) from the DOE ARM site at Manus Island $\left(2^{\circ} 3^{\prime} \mathrm{S}, 147^{\circ} 25^{\prime} \mathrm{E} ; 4 \mathrm{~m}\right.$ altitude). (b) Accumulation, daily, 2-day, and 5-day precipitation distributions calculated using $1 \mathrm{~h}$ data (1950-2013) from the Miami International Airport station $\left(25^{\circ} 80^{\prime} \mathrm{N}, 279^{\circ} 70^{\prime} \mathrm{E} ; 10.7 \mathrm{~m}\right.$ altitude) that is part of the NOAA/NCEI Climate Data Online System. (c) Accumulation, 3-h, 12-h, and daily precipitation distributions calculated from a 100-yr integration of the model with on-off precipitation. (d) Accumulation, daily, 2-day, and 5-day precipitation distributions calculated from a 200-yr integration of the model with on-off precipitation. In all cases we superimpose fits of the form (2) or (4) as appropriate, with distribution parameters calculated following appendix A. Note the different $x$ and $y$ axes in the left vs the right columns. Parameters were chosen to give similar accumulation and duration moment ratios as observations, resulting in the same set of parameters in both cases: $R_{0}=9 \mathrm{~mm} \mathrm{~h}^{-1}, D_{P}=17 \mathrm{~mm} \mathrm{~h}^{-1}, E=$ $0.1 \mathrm{~mm} \mathrm{~h}^{-1}, \bar{C}=+0.2 \mathrm{~mm} \mathrm{~h}^{-1}, D_{E}=3 \mathrm{~mm} \mathrm{~h}^{-1 / 2}$, and $b=0.2 \mathrm{~mm}$. All model parameters are also listed in Table S1.

similar accumulation and duration moment ratios compared to Miami Airport observations (see caption), and we use a value of $\bar{C}=0.2 \mathrm{~mm} \mathrm{~h}^{-1}$ adequate for mean moisture convergent conditions. No further attempts to tune the model were made. To make the output more consistent with observations, we integrate the model for 200 years (for consistent statistics) using a time step of $1 \mathrm{~min}$ and from this we calculate hourly precipitation values, which are used as the basis to calculate the remaining statistics. With some differences, Fig. 6d shows good qualitative agreement with observations. The model does generate PDFs well fitted by gamma distributions, and exhibits a decrease in the power-law exponent with increasing averaging interval, with cutoffs in line with observations.

The decrease in power-law exponent with increasing averaging interval $t_{\text {avg }}$ can be explained by revisiting section 4 , as well as by inspecting (18). As $t_{\text {avg increases }}$ the number of events distribution (analogous to the daily number of events distribution in Fig. 3b) is weighted toward more events per interval (simply reflecting the fact that there are more precipitating events in a month than 
in a day), which increases the contribution of the less asymmetric conditional precipitation distributions $p_{n}$, (13), in (14) $\left(p_{P}=\sum_{n=1}^{n_{\max }} w_{n} p_{n}\right)$. This shifts the resulting probability from small to larger values, flattening the power-law range, resulting in a smaller $\tau_{P}$ value, and even a negative $\tau_{P}$ for long enough averaging interval. At this point it would be more convenient to return to the usual gamma distribution definition in (1), but for consistency with the rest of the paper we continue using $\tau_{P}$ and $P_{L}$ (keeping in mind the translation between (1) and (2), $\theta=P_{L}$, and $k=1-\tau_{P}$ ). The relatively small increase of $P_{L}$ with averaging interval in the model occurs because the ratio $\sigma_{w}^{2} / \bar{w}$ [which is the main control on $P_{L}$ for different $t_{\text {avg }}$ in (17)] increases slowly with increasing $t_{\mathrm{avg}}$. This is discussed in more detail below.

\section{ASYMPTOTIC DYNAMICS FOR LONG AVERAGES}

We showed in Fig. 4 that the daily precipitation cutoff is set by the storm accumulation cutoff. As the average interval increases the effects of the aggregation of different storm accumulations start shifting the balance of terms that determines the value of $\hat{P}_{L}$ in (17). The temporal aggregation of storms starts dominating $\hat{P}_{L}$ when

$$
\bar{w} \gtrsim \frac{\sigma_{w}^{2}}{\bar{w}}+\frac{s_{L}}{2 \bar{s}} .
$$

At this point $\hat{P}_{L}$ can no longer be interpreted as a cutoff, but rather simply as a scale parameter, and $\hat{\tau}_{P}<0$. How short the averaging interval must be for (20) to hold depends on local climate conditions. For a given $s_{L}$, regions characterized by mean moisture convergence will tend to satisfy the inequality faster. This can be observed in Figs. 7a,b where we show the values of $\hat{P}_{L}$ and $\hat{\tau}_{P}$ calculated from several integrations of (5)-(7), together with their analytical approximations in (17) and (18), for precipitation averages ranging from 1 to 180 days. Two cases are shown representative of mean moisture divergent and convergent conditions: $E+\bar{C}=0$ and $0.3 \mathrm{~mm} \mathrm{~h}^{-1}$, respectively, all else being equal. For the two cases shown here the inequality is satisfied for a 26 day average in the mean moisture divergent case, and for a 4 day average in the mean moisture convergent case (Fig. 7b). That is, we expect roughly similar shapes of the distribution for averaging intervals on the order of a month in dry areas compared to averaging intervals on the order of days in wet areas.

From the central limit theorem (see von Storch and Zwiers 1999) we expect that $\sigma_{w} / \bar{w} \sim 1 / \bar{w}^{1 / 2}$ as $\bar{w}$ increases. That is, the number of precipitating events becomes more certain and localized around $\bar{w}$ as $t_{\text {avg }}$ increases (Fig. S10).
This implies that $\sigma_{w}^{2} / \bar{w}$ tends to a constant for long enough $t_{\text {avg }}$, which according to (17) implies that $\hat{P}_{L}$ eventually saturates and no longer keeps increasing with increases in averaging interval. This process occurs faster for mean moisture convergent regions (Fig. 7) as it depends on the mean number of events $\bar{w}$. In the limit where $\sigma_{w}^{2} / \bar{w}$ is constant, it can be shown from (18) that

$$
\hat{\tau}_{P} \propto-\bar{w} \propto-t_{\text {avg }} .
$$

Indeed $\hat{\tau}_{P}$ and the mean number of precipitating events $\bar{w}$ in $t_{\text {avg }}$ are very well anticorrelated. This explains how fast $\tau_{P}$ decreases for the mean moisture convergent region case (Fig. $7 b$ ). We note that as $\bar{w} \rightarrow \infty$ then $\hat{\tau}_{P} \rightarrow-\infty$ and the gamma distribution tends to a Gaussian (Thom 1958; Ropelewski et al. 1985), as might be expected from the central limit theorem. This transition to a Gaussianity process occurs faster for wet regions.

While these conclusions have been made in basis of the simple model, it should be emphasized that they have corresponding behavior in observations. Figures $7 \mathrm{c}$ and $7 \mathrm{~d}$ show similar plots for $\hat{P}_{L}$ and $\hat{\tau}_{P}$, respectively, as a function of averaging interval for 64 years of Miami Airport hourly precipitation data. We observe qualitatively similar curves, with $\hat{P}_{L}$ increasing with averaging interval, and apparently saturating for long-enough $t_{\mathrm{avg}}$ (although this occurs more slowly with $t_{\text {avg }}$ than in the model). The parameter $\hat{\tau}_{P}$ also decreases almost linearly with averaging interval. We note that the parameters of the model were not tuned to match this particular set of observations, which may explain some of the discrepancy in the value of $\hat{P}_{L}$ compared to observations. Typically across the parameter space, $\sigma_{w}^{2} / \bar{w}$ tends to a constant faster in the model compared to observations, which contributes to $\hat{P}_{L}$ saturating for smaller values in the model.

\section{Conclusions and discussion}

There is a long tradition of using gamma-like distributions to represent temporally averaged precipitation distributions. There has been little justification for their use beyond being a distribution bounded by zero that can represent skewed data (Ropelewski et al. 1985; Wilks and Eggleston 1992). Here we present a more fundamental view on how gamma-like distributions arise as a good fit to represent precipitation PDFs. To address this question, we use two simple stochastic models that, despite their simplicity, condense what is arguably the most important aspect that explains observed precipitation distributions-the competition in the moisture budget between fluctuations by moisture 

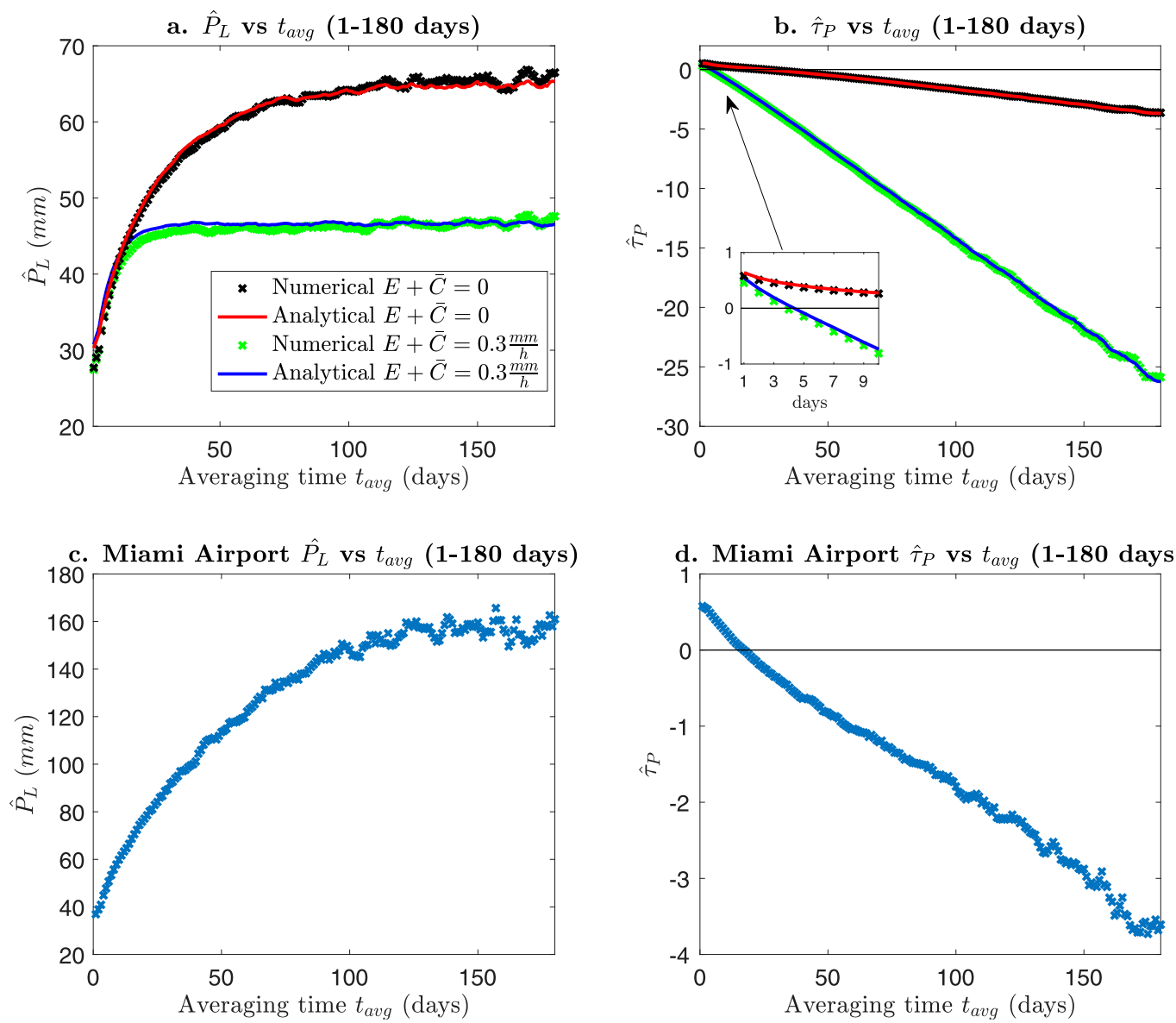

FIG. 7. Gamma distribution parameters (estimated using the method of moments) as a function of averaging interval $t_{\text {avg. }}$ (top) Estimations of (a) $\hat{P}_{L}$ and (b) $\hat{\tau}_{P}$ for different model runs (with an integration time step of 1 min) for $t_{\text {avg }}$ ranging from 1 to 180 days. Two sets of runs are shown, one under moisture-convergent conditions $\left(\bar{C}=+0.2 \mathrm{~mm} \mathrm{~h}^{-1}\right)$ and one under moisture-divergent conditions $\left(\bar{C}=-0.1 \mathrm{~mm} \mathrm{~h}^{-1}\right)$, with $E=0.1 \mathrm{~mm} \mathrm{~h}^{-1}$. The remaining model parameters are $R_{0}=10 \mathrm{~mm} \mathrm{~h}^{-1}, D_{P}=15 \mathrm{~mm} \mathrm{~h}^{-1 / 2}, D_{E}=3 \mathrm{~mm} \mathrm{~h}^{-1 / 2}$, and $b=1 \mathrm{~mm}$. All model parameters are also listed in Table S1. To reduce fluctuations, especially for longer $t_{\text {avg }}$, in each case estimations for 10 independent 100-yr runs are averaged and then a 5-day running mean is applied [except for inset in (b)]. (bottom) Miami Airport observations (1950-2013) of (5-day running mean) (c) $\hat{P}_{L}$ and (d) $\hat{\tau}_{P}$ as a function of $t_{\text {avg }}$ using hourly precipitation as the basis.

convergence and dissipation by moisture loss due to precipitation. Under this simplified framework, gammalike distributions arise by the interplay between the distribution of storm accumulations (from event onset to termination) and the distribution of the number of these storms (number of events distribution) in the averaging interval of interest. The distribution of accumulations can be physically derived from the moisture equation, a fundamental equation of atmospheric models (SN14; N17), with the distribution shape consisting of a relatively sharp power-law range with an exponential cutoff $s_{L}$ for large sizes. Here we extend the insight gained from the study of accumulations to temporally averaged precipitation distributions by noting that the total precipitation falling in the temporal average of interest (for instance a day) basically consists in the addition of different accumulation events occurring within this one day period (or other $t_{\mathrm{avg}}$ ). This leads to higher probabilities of larger values and smaller probabilities for smaller values with respect to accumulations, yielding power-law exponents for daily precipitation distributions that are strictly smaller than the underlying accumulations. In addition, the resulting daily precipitation distribution also features an exponential cutoff, with properties inherited from the underlying accumulations. This results in a daily precipitation PDF that can be well fitted by gamma distributions.

There are several implications arising from this framework. From previous research (SN14; N17) we know that the accumulation distribution cutoff is proportional to the 
size of moisture convergence fluctuations in the precipitating regime $\left(s_{L} \propto D_{P}\right)$. Here we show that this statement can also be made for daily precipitation (or similar $t_{\text {avg }}$ ). That is, the daily precipitation cutoff is also proportional to the size of moisture convergence fluctuations $\left(P_{L} \propto s_{L} \propto D_{P}\right)$. The proportionality between $s_{L}$ and $P_{L}$ has been observed to occur over the United States (MN18), and this framework provides explanation for this observational result.

The importance of shifts in the accumulation cutoff has been shown in general circulation models (N17) and observations (MN18). These shifts in cutoff, proportional to changes in $D_{P}$, involve increases in moisture (thermodynamic contribution) and changes in convergence (dynamic contribution) (Pfahl et al. 2017; Norris et al. 2019b) - note that both effects enter into the same parameter of the PDF. In most regions this implies an increase in $s_{L}$, which extends the accumulation powerlaw range, yielding approximately exponential increases in the probability of the largest accumulations (N17; Norris et al. 2019a). One of the main conclusions of this work is that a similar increase in probability of daily (or similar averages) precipitation extremes occurs as moisture increases. In this case the changes in probability will be slightly more complicated than for accumulations, as the power-law exponent will also change as $D_{P}$ increases [see (18)], and also depends on changes of the dry regime dynamics.

To illustrate this, we consider the effect that a postulated increase in the amplitude of fluctuations of moisture convergence under a global warming scenario has on daily precipitation distributions. Figure 8 shows the risk ratio (Otto et al. 2012; N17), defined as the ratio of the probability (conditioned on event occurrence) of daily precipitation larger than a certain amount ( $x$ axis in Fig. 8 ) in the warmer compared to current conditions, for two different cases calculated using long runs of the ramp precipitation model. In the first case (red) we consider a $21 \%$ increase of $D_{P}$ and $D_{E}$, which would correspond to a Clausius-Clapeyron scaling with $3^{\circ} \mathrm{C}$ warming (increase of $3 \times 7 \%$ ) in the amplitude of moisture convergence fluctuations. In the second case (blue), only $D_{P}$ scales up. This case can provide insight into the effect of changes in vertical velocity (which are linked to changes in the dynamic contribution to moisture convergence via the continuity equation) that are asymmetric for ascending and descending regimes that have been suggested to occur under global warming (Pendergrass and Gerber 2016). In both cases, increases in $D_{P}$ yield increases in the daily precipitation cutoff $P_{L}$ with resulting exponential increases in the probability of the largest daily precipitation values, much as it occurs for accumulations. Similar increases in risk ratio for extreme daily precipitation have been

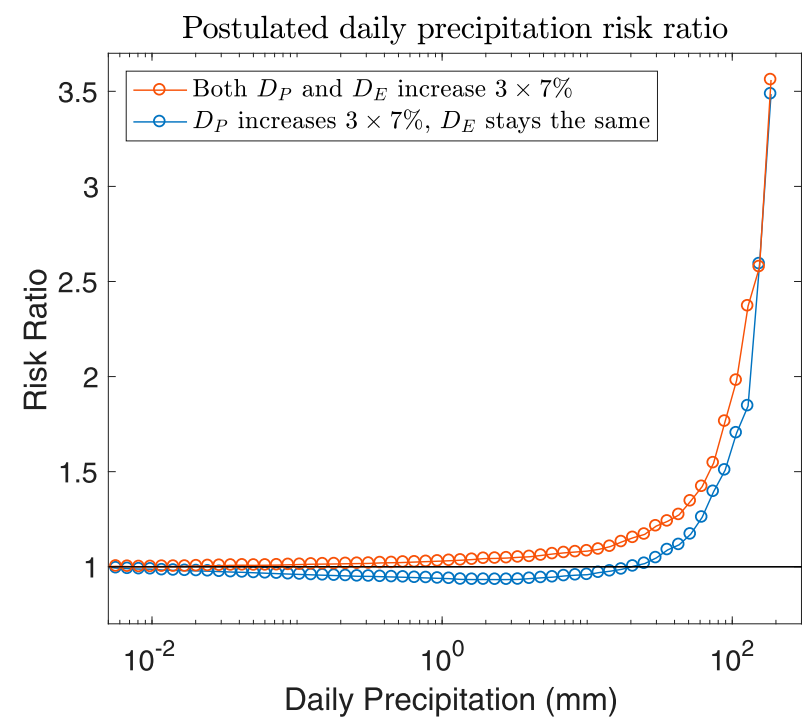

FIG. 8. Risk ratio $R(x)$, calculated as $R(x)=\int_{x}^{\infty} p_{P}^{\mathrm{GW}} d P / \int_{x}^{\infty} p_{P}^{\text {Hist }} d P$, where $p_{P}^{\text {Hist }}$ is the daily precipitation distribution under current conditions and $p_{P}^{\mathrm{GW}}$ is the daily precipitation distribution under warmer conditions, from 1000-yr runs of the ramp precipitation model. Parameters are given in Table S1, with warmer conditions represented in case 1 (red) by both precipitating and nonprecipitating moisture convergence noise amplitude $D_{P}$ and $D_{E}$ scaled up by a factor of $(1+\gamma)$, where $\gamma=3 \times 0.07$, whereas in case 2 (blue) only $D_{P}$ scales. The calculated gamma distribution parameters in case 1 are $\hat{P}_{L}^{\mathrm{Hist}}=41 \mathrm{~mm}, \hat{P}_{L}^{\mathrm{GW}}=50 \mathrm{~mm}, \hat{\tau}_{P}^{\mathrm{Hist}}=0.75$, and $\hat{\tau}_{P}^{\mathrm{GW}}=0.75$, and in case 2 are $\hat{P}_{L}^{\mathrm{Hist}}=41 \mathrm{~mm}, \hat{P}_{L}^{\mathrm{GW}}=50 \mathrm{~mm}$, $\hat{\tau}_{P}^{\mathrm{Hist}}=0.75$, and $\hat{\tau}_{P}^{\mathrm{GW}}=0.79$.

observed in the United States during recent decades (MN18). The main difference between the two cases is in how the power-law exponents adjust, which points to the role of the dry regime dynamics. In the more symmetric first case-with increases in both $D_{P}$ and $D_{E}$ - the power-law exponents are similar in current and warmer conditions (as is the case for accumulations), resulting in exponential increases in risk ratio starting in the moderate daily precipitation range. In the second case $\tau_{P}$ adjusts, increasing its value for warmer conditions. This results in a reduction in the probability of moderate daily precipitation, with exponential increases in risk ratio starting for larger values. We argue that the simple arguments laid out here may account for changes in the occurrences of extremes in the daily precipitation distribution that have already been observed (e.g., Kunkel et al. 2013; MN18) or that have been projected to occur for climate warming scenarios (Fischer and Knutti 2016; Pendergrass 2018).

The simple scaling argument $\left(P_{L} \propto D_{P}\right)$ also provides explanation for other relations regarding daily precipitation extremes found in the literature. Several studies have shown the scale parameter (our $P_{L}$ ) of gamma distributions to be a useful indicator of changes in daily 
precipitation extremes (Groisman et al. 1999; Wilby and Wigley 2002; Watterson and Dix 2003; MN18). Our analytical results, (17), provide a more formal justification of this, and suggest that changes in $P_{L}$ may also be used to track changes in extremes for other relatively short averages. Another aspect that may be explained by this simple framework is the increase in daily precipitation variability observed in global warming model projections (Pendergrass et al. 2017). Not only does $P_{L}$ scale with moisture availability in our model, but our results also imply a similar scaling for daily precipitation variance $\sigma_{P}^{2}$ [see (10) and (16)]. Increases in $\sigma_{P}^{2}$ occur primarily by increases in storm accumulation variance at daily scales, although increases in the variability in the number of storms, (16), can also enter into play, especially at longer time scales. The relative contribution to these changes could be evaluated in a climate model.

Overall, for daily precipitation (or other relatively short temporal average, as elaborated below) the wet regime controls to a great extent the resulting time-average intensity distribution. Changes in the dry regime (with wet regime fixed) modify the resulting distribution to a secondary extent, with slight adjustments to the power-law exponent. This dependence on the dry regime, encapsulated in changes in mean moisture convergence $\bar{C}$ (or $D_{E}$ ), may be relevant to explain changes in the distribution under global warming in subtropicalmidlatitude transition zones (e.g., Garreaud et al. 2017; Swain et al. 2018), that may occur in association to a poleward expansion of subtropical high pressure regions (Lu et al. 2007; Frierson et al. 2007; Kang and Lu 2012; Levine and Schneider 2015). All else fixed, regions characterized by mean moisture convergence have a gentler power-law range compared to regions characterized by mean moisture divergence in the model.

Although we focus on daily precipitation, the framework presented here applies to other averaging intervals as well. For shorter, subdaily averaging intervals, caution is required when there is not a good separation between the averaging interval $t_{\text {avg }}$ and the event duration cutoff $t_{L}$. The computation using mixture distributions that explains the resulting gamma-like distributions in section 4 , and the analytical approximations for $\hat{\tau}_{P}$ and $\hat{P}_{L},(17)$ and (18), may become poor approximations for short averages (as $t_{\mathrm{avg}} \sim t_{L}$ ). However, the numerical stochastic model generates subdaily distributions that resemble observations (Figs. 6a,c), and this suggests a smooth evolution of behavior with averaging interval. In all cases there is a tendency for lower values of $\hat{\tau}_{P}$ as the averaging interval $t_{\text {avg }}(3-, 12-, 24-\mathrm{h})$ increases. Thus, the qualitative rationale for the relationship of the gamma distribution to the accumulation distribution continues to work over some range of subdaily time scales (which may have a regional dependence because $t_{L}$ can vary).

For longer averaging intervals, $t_{\mathrm{avg}} \gg t_{L}$ (i.e., much longer than the precipitation duration cutoff, which tends to occur for daily or longer averages), we can demonstrate that the daily precipitation power-law exponent is strictly smaller in magnitude than the accumulation power-law exponent. Because it typically rains more than once per averaging interval (Fig. 3b), the precipitation intensity distribution, (14), as a mixture of the conditional distributions given by (13), will have less probability than accumulations for small values, and more probability for larger values, yielding a less steep precipitation intensity power-law range than for accumulations. Similarly, longer averages will have a number of events distribution weighted to a larger number of events within $t_{\text {avg }}$, yielding power-law exponents that eventually change sign for sufficiently long averages. This argument leads us to conclude that $\hat{\tau}_{P}$ decreases monotonically with averaging interval $t_{\text {avg }}$ (giving allowance to sampling variations in observations). In fact, for long enough $t_{\mathrm{avg}}$, we show that $-\hat{\tau}_{P}$ is linearly proportional to the mean number of events $\bar{w}$ per averaging interval $t_{\mathrm{avg}}$.

For regions with plentiful moisture supply, the paradigm of thinking about a power law and cutoff for the time-average precipitation distribution applies up to an averaging interval measured in days, with $P_{L}$ scaling with moisture. For much longer averaging interval, the resulting distribution begins to have properties not much different from a Gaussian, in which case changes in the mean and variance, (15) and (16), may be more useful. In mean moisture divergence regions the power-law and cutoff paradigm may be valid for longer $t_{\text {avg }}$ (on the order of a month), as the power-law exponent decreases slowly due to the mean number of precipitating events $\bar{w}$ being considerable smaller in this case. Thus, the framework presented here allows a window into the evolution of the precipitation distribution as a function of different time scales and climate conditions.

Acknowledgments. This research was supported by National Science Foundation Grant AGS-1540518 and by National Oceanic and Atmospheric Administration Grant NA18OAR4310280. Manus Island precipitation data are courtesy of the U.S. Department of Energy Atmospheric Radiation Measurement (ARM) Climate Research Facility Tropical West Pacific field campaign. We thank K. Schiro for assistance with this dataset. Miami Airport, Hartford Airport and Fullerton Dam precipitation data are courtesy of the NOAA/NCEI Climate Data Online system (https://www.ncdc.noaa.gov/ cdo-web/search?datasetid=PRECIP_HLY $\backslash$ \#). We thank F. Zwiers for a postseminar question that helped motivate 
this work and J. Meyerson for graphical assistance. A portion of this work has previously been presented at an American Physical Society meeting (Martinez-Villalobos and Neelin 2018a) and at an American Geophysical Union meeting (Martinez-Villalobos and Neelin 2018c).

\section{APPENDIX A}

\section{Estimation of Accumulation and Temporally Averaged Precipitation Distribution Parameters}

To estimate parameters of the distributions in Figs. 2 and 6 we use a simple multivariate linear regression of the binned probabilities, which we assume have a shape $p_{x} \sim x^{-\tau_{x}} \exp \left(-x / x_{L}\right)$, onto the accumulation or temporally averaged precipitation sizes (represented as $x$ here). The parameters are calculated by assuming a relationship between $\log (p)$ and functions of $x$ as follows:

$$
\log (p)=c_{1}+c_{2} \log (x)+c_{3} x
$$

and regressing to estimate the $c_{i}$ coefficients. The parameters are then given as $\tau_{x}=-c_{2}$, and $x_{L}=-\left(1 / c_{3}\right)$.

This way to calculate parameters is consistent for both accumulations and daily precipitation distributions and generally give more consistent fits for variation in data resolution. We note that this methodology has a small dependence on the binning scheme used. The parameters calculated in this way are proportional to parameters calculated using either maximum likelihood or the method of moments (Fig. S1).

\section{APPENDIX B}

\section{Distribution of Wet- and Dry-Spell Durations}

The distribution of event durations $p_{t}$, that informs the range of validity of the analytic approximations, is derived in SN14 for the case of on-off precipitation as a rescaling of the accumulation distribution, (9):

$$
p_{t}=\frac{\bar{t}}{\sqrt{\pi t_{L}}} \exp \left(\frac{2 \bar{t}}{t_{L}}\right) \exp \left(-\frac{\bar{t}^{2}}{t_{L} t}\right) \exp \left(-\frac{t}{t_{L}}\right) t^{-3 / 2},
$$

with $t$ the event duration. The mean duration $\bar{t}$ and the duration cutoff $t_{L}$ are given by

$$
\bar{t}=\frac{b}{R_{0}}, \quad t_{L}=\frac{2 D_{P}^{2}}{R_{0}^{2}} .
$$

The analytical solution for the distribution of event durations in the ramp precipitation case can be adapted from similar equations in the finance literature (Yi 2010), discussed in section 2. An important point of this solution is that $t_{L}=1 / \alpha$, which is used to numerically validate (11) in this case.

The solution for the distribution of dry-spell duration (SN14) has the same shape as (B1), but with mean dryspell duration $\bar{t}_{D}=b /(E+\bar{C})$ and dry-spell duration cutoff $t_{L}^{D}=2 D_{E}^{2} /(E+\bar{C})^{2}(E+\bar{C} \geq 0)$.

\section{APPENDIX C}

\section{Analytical Formulas for $p_{n}$ Distributions and Moments}

The starting point is to note that the accumulation distribution given by (9) can be rewritten in a more standard form for inverse Gaussians as

$$
p_{s}(\mu, \lambda)=\sqrt{\frac{\lambda}{2 \pi s^{3}}} \exp \left[-\frac{\lambda(s-\mu)^{2}}{2 \mu^{2} s}\right],
$$

with $\lambda=2 \bar{s}^{2} / s_{L}$ and $\mu=\bar{s}$. If, as in (12), we sample a value $s_{i}$ from the same $p_{s}$ distribution $n$ times, then the variable $P_{n}=\sum_{i}^{n} s_{i}$ is distributed as $p_{s}\left(n \mu, n^{2} \lambda\right)$, which is a particular case of a more general additive property of inverse Gaussians (Tweedie 1957; Folks and Chhikara 1978). Going back to the original variables, we find that $P_{n}$ is distributed as in (13).

The mean and variance of an inverse Gaussian such as $(\mathrm{C} 1)$ are given by $\bar{s}=\mu$, and $\sigma_{s}^{2}=\left(\mu^{3} / \lambda\right)=(1 / 2) \bar{s}_{L}$. From this we find that the mean $\left\langle P_{n}\right\rangle$ and variance $\left\langle P_{n}^{2}\right\rangle-\left\langle P_{n}^{2}\right\rangle$ of the $p_{n}$ distribution are given by

$$
\begin{aligned}
\left\langle P_{n}\right\rangle & =n \bar{s}, \\
\left\langle P_{n}^{2}\right\rangle-\left\langle P_{n}^{2}\right\rangle & =n \sigma_{s}^{2}=\frac{n}{2} \bar{s} S_{L} .
\end{aligned}
$$

\section{APPENDIX D}

\section{Derivation of Gamma Distribution Parameter Formulas}

The $m$ th moment about zero $\left\langle P^{m}\right\rangle$ of a distribution $p_{p}$ is given by

$$
\left\langle P^{m}\right\rangle=\int_{0}^{\infty} P^{m} p_{P} d P
$$

Here $p_{p}$ is a temporally averaged precipitation distribution. Using (14), this can be rewritten in terms of the moments of the $p_{n}$ distributions, (13), which we denote as $\left\langle P_{n}^{m}\right\rangle$, as 


$$
\begin{aligned}
\left\langle P^{m}\right\rangle & =\int_{0}^{\infty} P^{m} \sum_{n=1}^{n_{\max }} w_{n} p_{n} d P=\sum_{n=1}^{n_{\max }} w_{n} \int_{0}^{\infty} P^{m} p_{n} d P \\
& =\sum_{n=1}^{n_{\max }} w_{n}\left\langle P_{n}^{m}\right\rangle .
\end{aligned}
$$

From this we find, using $(\mathrm{C} 2)$,

$$
\bar{P}=\langle P\rangle=\sum_{n=1}^{n_{\max }} w_{n}\left\langle P_{n}\right\rangle=\bar{s} \sum_{n=1}^{n_{\max }} n w_{n}=\bar{w} \bar{s}
$$

(note that $\bar{w}=\sum_{n=1}^{n_{\max }} n w_{n}$ ), which is the same as (15).

The demonstration of (16) goes along the same lines:

$$
\sigma_{P}^{2}=\left\langle P^{2}\right\rangle-\langle P\rangle^{2}=\sum_{n=1}^{n_{\max }} w_{n}\left\langle P_{n}^{2}\right\rangle-\left(\sum_{n=1}^{n_{\max }} w_{n}\left\langle P_{n}\right\rangle\right)^{2} .
$$

Using (C2) and (C3), this can be rewritten as

$$
\begin{aligned}
\sigma_{P}^{2} & =\sum_{n=1}^{n_{\max }} w_{n}\left(n \sigma_{s}^{2}+n^{2} \bar{s}^{2}\right)-\bar{s}^{2}\left(\sum_{n=1}^{n_{\max }} n w_{n}\right)^{2} \\
& =\bar{w} \sigma_{s}^{2}+\bar{s}^{2}\left[\sum_{n=1}^{n_{\max }} n^{2} w_{n}-\left(\sum_{n=1}^{n_{\max }} n w_{n}\right)^{2}\right] .
\end{aligned}
$$

Noting that $\sigma_{w}^{2}=\sum_{n=1}^{n_{\max }} n^{2} w_{n}-\left(\sum_{n=1}^{n_{\max }} n w_{n}\right)^{2}$, then we arrive at expression (16).

We calculate $\hat{P}_{L}$ and $\hat{\tau}_{P}$ formulas using the method of moments yielding

$$
\begin{aligned}
\bar{P} & =\left(1-\hat{\tau}_{P}\right) \hat{P}_{L}, \\
\sigma_{P}^{2} & =\left(1-\hat{\tau}_{P}\right) \hat{P}_{L}^{2} .
\end{aligned}
$$

Noting that $s_{L}=2 \sigma_{s}^{2} / \bar{s}$ (SN14), this can be rearranged to yield (17) and (18).

\section{REFERENCES}

Abbott, T. H., S. N. Stechmann, and J. D. Neelin, 2016: Long temporal autocorrelations in tropical precipitation data and spike train prototypes. Geophys. Res. Lett., 43, 11 472-11480, https://doi.org/10.1002/2016GL071282.

Ahmed, F., and C. Schumacher, 2015: Convective and stratiform components of the precipitation-moisture relationship. Geophys. Res. Lett., 42, 10 453-10 462, https://doi.org/10.1002/ 2015 GL066957.

_ , and J. D. Neelin, 2019: Explaining scales and statistics of tropical precipitation clusters with a stochastic model. J. Atmos. Sci., 76, 3063-3087, https://doi.org/10.1175/JAS-D-18-0368.1.

Barbero, R., H. J. Fowler, G. Lenderink, and S. Blenkinsop, 2017: Is the intensification of precipitation extremes with global warming better detected at hourly than daily resolutions? Geophys. Res. Lett., 44, 974-983, https://doi.org/10.1002/ 2016GL071917.
Bretherton, C. S., M. E. Peters, and L. E. Back, 2004: Relationships between water vapor path and precipitation over the tropical oceans. J. Climate, 17, 1517-1528, https://doi.org/10.1175/15200442(2004)017<1517:RBWVPA > 2.0.CO;2.

Cho, H.-K., K. P. Bowman, and G. R. North, 2004: A comparison of gamma and lognormal distributions for characterizing satellite rain rates from the Tropical Rainfall Measuring Mission. J. Appl. Meteor., 43, 1586-1597, https:// doi.org/10.1175/JAM2165.1.

Deluca, A., and Á. Corral, 2014: Scale invariant events and dry spells for medium-resolution local rain data. Nonlinear Processes Geophys., 21, 555-567, https://doi.org/10.5194/ npg-21-555-2014.

Ewald, B., and C. Penland, 2009: Numerical generation of stochastic differential equations in climate models. Handbook of Numerical Analysis, Vol. 14, 279-306, https://doi.org/10.1016/ S1570-8659(08)00206-8.

Fischer, E. M., and R. Knutti, 2016: Observed heavy precipitation increase confirms theory and early models. Nat. Climate Change, 6, 986-991, https://doi.org/10.1038/nclimate3110.

Folks, J. L., and R. S. Chhikara, 1978: The inverse Gaussian distribution and its statistical application-A review. J. Roy. Stat. Soc., 40B, 263-289, https://www.jstor.org/stable/ 2984691.

Frierson, D. M. W., J. Lu, and G. Chen, 2007: Width of the Hadley cell in simple and comprehensive general circulation models. Geophys. Res. Lett., 34, L18804, https://doi.org/10.1029/ 2007 GL031115.

Frühwirth-Schnatter, S., 2006: Finite Mixture and Markov Switching Models. 1st ed. Springer-Verlag, 494 pp., https://doi.org/10.1007/ 978-0-387-35768-3.

Gardiner, C. W., 2009: Stochastic Methods: A Handbook for the Natural and Social Sciences. Springer, $447 \mathrm{pp}$.

Garreaud, R. D., and Coauthors, 2017: The 2010-2015 megadrought in central Chile: Impacts on regional hydroclimate and vegetation. Hydrol. Earth Syst. Sci., 21, 6307-6327, https://doi.org/ 10.5194/hess-21-6307-2017.

Gaustad, K., and L. Riihimaki, 1996: MWR retrievals (MWRRET1LILJCLOU), 1998-01-01 to 2010-12-31, Tropical Western Pacific (TWP) Central Facility, Manus I., PNG (C1) (updated hourly). ARM Climate Research Facility Data Archive, https://doi.org/10.5439/1027369.

Groisman, P. Y., and Coauthors, 1999: Changes in the probability of heavy precipitation: Important indicators of climatic change. Climatic Change, 42, 243-283, https://doi.org/10.1023/ A:1005432803188.

Holdridge, D., and J. Kyrouac, 1997: Surface meteorological instrumentation (MET), 1998-01-01 to 2010-12-31, Tropical Western Pacific (TWP) Central Facility, Manus I., PNG (C1) (updated hourly). ARM Climate Research Facility Data Archive, https://doi.org/10.5439/1025220.

Hottovy, S., and S. N. Stechmann, 2015: A spatiotemporal stochastic model for tropical precipitation and water vapor dynamics. J. Atmos. Sci., 72, 4721-4738, https://doi.org/10.1175/JAS-D15-0119.1.

Husak, G. J., J. Michaelsen, and C. Funk, 2007: Use of the gamma distribution to represent monthly rainfall in Africa for drought monitoring applications. Int. J. Climatol., 27, 935-944, https:// doi.org/10.1002/joc.1441.

Ison, N. T., A. M. Feyerherm, and L. D. Bark, 1971: Wet period precipitation and the gamma distribution. J. Appl. Meteor., 10, 658-665, https://doi.org/10.1175/1520-0450(1971)010<0658: WPPATG $>2.0 . \mathrm{CO} ; 2$. 
Kang, S. M., and J. Lu, 2012: Expansion of the Hadley cell under global warming: Winter versus summer. J. Climate, 25, 83878393, https://doi.org/10.1175/JCLI-D-12-00323.1.

Katz, R. W., 1977: Precipitation as a chain-dependent process. J. Appl. Meteor., 16, 671-676, https://doi.org/10.1175/15200450(1977)016<0671:PAACDP>2.0.CO;2.

Kirchmeier-Young, M. C., D. J. Lorenz, and D. J. Vimont, 2016: Extreme event verification for probabilistic downscaling. J. Appl. Meteor. Climatol., 55, 2411-2430, https://doi.org/ 10.1175/JAMC-D-16-0043.1.

Kunkel, K. E., and Coauthors, 2013: Monitoring and understanding trends in extreme storms: State of knowledge. Bull. Amer. Meteor. Soc., 94, 499-514, https://doi.org/10.1175/BAMSD-11-00262.1.

Kuo, Y.-H., K. A. Schiro, and J. D. Neelin, 2018: Convective transition statistics over tropical oceans for climate model diagnostics: Observational baseline. J. Atmos. Sci., 75, 15531570, https://doi.org/10.1175/JAS-D-17-0287.1.

Lenderink, G., and E. van Meijgaard, 2008: Increase in hourly precipitation extremes beyond expectations from temperature changes. Nat. Geosci., 1, 511-514, https://doi.org/10.1038/ ngeo262.

Levine, X. J., and T. Schneider, 2015: Baroclinic eddies and the extent of the Hadley circulation: An idealized GCM study. J. Atmos. Sci., 72, 2744-2761, https://doi.org/10.1175/JAS-D14-0152.1.

Lu, J., G. A. Vecchi, and T. Reichler, 2007: Expansion of the Hadley cell under global warming. Geophys. Res. Lett., 34, L06805, https://doi.org/10.1029/2006GL028443.

Martinez-Villalobos, C., and J. D. Neelin, 2018a: Precipitation accumulations, intensities and durations. APS March Meeting 2018, Los Angeles, CA, American Physical Society, R47.00001, https://meetings.aps.org/Meeting/MAR18/Session/R47.1.

$\longrightarrow$, and $-2018 \mathrm{~b}$ : Shifts in precipitation accumulation extremes during the warm season over the United States. Geophys. Res. Lett., 45, 8586-8595, https://doi.org/10.1029/2018GL078465.

- , and ——, 2018c: What sets the probability distribution of precipitation? 2018 Fall Meeting, Washington, DC, Amer. Geophys. Union, Abstract NG11A-04, https://agu.confex.com/ agu/fm18/meetingapp.cgi/Paper/416514.

Muller, C. J., L. E. Back, P. A. O'Gorman, and K. A. Emanuel, 2009: A model for the relationship between tropical precipitation and column water vapor. Geophys. Res. Lett., 36, L16804, https://doi.org/10.1029/2009GL039667.

Neelin, J. D., and N. Zeng, 2000: A quasi-equilibrium tropical circulation model-Formulation. J. Atmos. Sci., 57, 1741-1766, https://doi.org/10.1175/1520-0469(2000)057<1741:AQETCM> 2.0.CO;2.

- O. Peters, and K. Hales, 2009: The transition to strong convection. J. Atmos. Sci., 66, 2367-2384, https://doi.org/10.1175/ 2009JAS2962.1.

—, S. Sahany, S. N. Stechmann, and D. N. Bernstein, 2017: Global warming precipitation accumulation increases above the current-climate cutoff scale. Proc. Natl. Acad. Sci. USA, 114, 1258-1263, https://doi.org/10.1073/pnas.1615333114.

Norris, J., G. Chen, and J. D. Neelin, 2019a: Changes in frequency of large precipitation accumulations over land in a warming climate from the CESM Large Ensemble: The roles of moisture, circulation, and duration. J. Climate, 32, 5397-5416, https://doi.org/10.1175/JCLI-D-18-0600.1.

, — - and — , 2019b: Thermodynamic versus dynamic controls on extreme precipitation in a warming climate from the Community Earth System Model Large Ensemble.
J. Climate, 32, 1025-1045, https://doi.org/10.1175/JCLI-D-180302.1.

O'Gorman, P. A., 2014: Contrasting responses of mean and extreme snowfall to climate change. Nature, 512, 416-418, https://doi.org/ 10.1038/nature13625.

Otto, F. E. L., N. Massey, G. J. van Oldenborgh, R. G. Jones, and M. R. Allen, 2012: Reconciling two approaches to attribution of the 2010 Russian heat wave. Geophys. Res. Lett., 39, L04702, https://doi.org/10.1029/2011GL050422.

Papalexiou, S. M., and D. Koutsoyiannis, 2013: Battle of extreme value distributions: A global survey on extreme daily rainfall. Water Resour. Res., 49, 187-201, https://doi.org/10.1029/ 2012WR012557.

Pendergrass, A. G., 2018: What precipitation is extreme? Science, 360, 1072-1073, https://doi.org/10.1126/science.aat1871. , and E. P. Gerber, 2016: The rain is askew: Two idealized models relating vertical velocity and precipitation distributions in a warming world. J. Climate, 29, 6445-6462, https:// doi.org/10.1175/JCLI-D-16-0097.1.

_ , R. Knutti, F. Lehner, C. Deser, and B. M. Sanderson, 2017: Precipitation variability increases in a warmer climate. Sci. Rep., 7, 17966, https://doi.org/10.1038/s41598-017-17966-y.

Peters, O., and J. D. Neelin, 2006: Critical phenomena in atmospheric precipitation. Nat. Phys., 2, 393-396, https://doi.org/ 10.1038/nphys 314 .

_ C. Hertlein, and K. Christensen, 2001: A complexity view of rainfall. Phys. Rev. Lett., 88, 018701, https://doi.org/10.1103/ PhysRevLett.88.018701.

_- A. Deluca, A. Corral, J. D. Neelin, and C. E. Holloway, 2010: Universality of rain event size distributions. J. Stat. Mech., 2010, P11030, https://doi.org/10.1088/1742-5468/ 2010/11/P11030.

Pfahl, S., P. A. O'Gorman, and E. M. Fischer, 2017: Understanding the regional pattern of projected future changes in extreme precipitation. Nat. Climate Change, 7, 423-427, https://doi.org/ 10.1038/nclimate3287.

Prein, A. F., R. M. Rasmussen, K. Ikeda, C. Liu, M. P. Clark, and G. J. Holland, 2017: The future intensification of hourly precipitation extremes. Nat. Climate Change, 7, 48-52, https:// doi.org/10.1038/nclimate3168.

Quinn, K. M., and J. D. Neelin, 2017: Distributions of tropical precipitation cluster power and their changes under global warming. Part I: Observational baseline and comparison to a high-resolution atmospheric model. J. Climate, 30, 80338044, https://doi.org/10.1175/JCLI-D-16-0683.1.

Raymond, D. J., 2000: Thermodynamic control of tropical rainfall. Quart. J. Roy. Meteor. Soc., 126, 889-898, https://doi.org/ 10.1002/qj.49712656406.

Redner, S., 2001: A Guide To First-Passage Processes. Cambridge University Press, 312 pp., https://doi.org/10.1017/ CBO9780511606014.

Richardson, C. W., 1981: Stochastic simulation of daily precipitation, temperature, and solar radiation. Water Resour. Res., 17, 182-190, https://doi.org/10.1029/WR017i001p00182.

Ropelewski, C. F., J. E. Janowiak, and M. S. Halpert, 1985: The analysis and display of real time surface climate data. Mon. Wea. Rev., 113, 1101-1106, https://doi.org/10.1175/1520-0493(1985) $113<1101$ :TAADOR $>2.0 . \mathrm{CO} ; 2$.

Sahany, S., J. D. Neelin, K. Hales, and R. B. Neale, 2012: Temperature-moisture dependence of the deep convective transition as a constraint on entrainment in climate models. J. Atmos. Sci., 69, 1340-1358, https://doi.org/10.1175/JAS-D11-0164.1. 
and - 2014: Deep convective transition characteristics in the Community Climate System Model and changes under global warming. J. Climate, 27, 9214-9232, https://doi.org/10.1175/JCLI-D-13-00747.1.

Schiro, K. A., J. D. Neelin, D. K. Adams, and B. R. Lintner, 2016: Deep convection and column water vapor over tropical land versus tropical ocean: A comparison between the Amazon and the tropical western Pacific. J. Atmos. Sci., 73, 4043-4063, https://doi.org/10.1175/JAS-D-16-0119.1.

Schleiss, M., 2018: How intermittency affects the rate at which rainfall extremes respond to changes in temperature. Earth Syst. Dyn., 9, 955-968, https://doi.org/10.5194/esd-9-955-2018.

Seager, R., and N. Henderson, 2013: Diagnostic computation of moisture budgets in the ERA-Interim reanalysis with reference to analysis of CMIP-archived atmospheric model data. J. Climate, 26, 7876-7901, https://doi.org/10.1175/JCLI-D-1300018.1.

Sobel, A., and E. Maloney, 2013: Moisture modes and the eastward propagation of the MJO. J. Atmos. Sci., 70, 187-192, https:// doi.org/10.1175/JAS-D-12-0189.1.

Stechmann, S. N., and J. D. Neelin, 2014: First-passage-time prototypes for precipitation statistics. J. Atmos. Sci., 71, 3269-3291, https://doi.org/10.1175/JAS-D-13-0268.1.

Swain, D. L., B. Langenbrunner, J. D. Neelin, and A. Hall, 2018: Increasing precipitation volatility in twenty-first-century California. Nat. Climate Change, 8, 427-433, https://doi.org/ 10.1038/s41558-018-0140-y.

Thom, H. C. S., 1958: A note on the gamma distribution. Mon Wea. Rev., 86, 117-122, https://doi.org/10.1175/1520-0493(1958) 086<0117:ANOTGD $>2.0 . \mathrm{CO} ; 2$.
Tweedie, M. C. K., 1957: Statistical properties of inverse Gaussian distributions. I. Ann. Math. Stat., 28, 362-377.

von Storch, H., and F. W. Zwiers, 1999: Statistical Analysis in Climate Research. Cambridge University Press, 484 pp., https://doi.org/10.1017/CBO9780511612336.

Watterson, I. G., and M. R. Dix, 2003: Simulated changes due to global warming in daily precipitation means and extremes and their interpretation using the gamma distribution. J. Geophys. Res., 108, 4379, https://doi.org/10.1029/ 2002JD002928.

Westra, S., and Coauthors, 2014: Future changes to the intensity and frequency of short-duration extreme rainfall. Rev. Geophys., 52, 522-555, https://doi.org/10.1002/2014RG000464.

Wilby, R. L., and T. M. L. Wigley, 2002: Future changes in the distribution of daily precipitation totals across North America. Geophys. Res. Lett., 29, 1135, https://doi.org/10.1029/ 2001 GL013048.

Wilks, D. S., 1995: Statistical Methods in the Atmospheric Sciences: An Introduction. Academic Press, $467 \mathrm{pp}$

_, and K. L. Eggleston, 1992: Estimating monthly and seasonal precipitation distributions using the 30- and 90-day outlooks. J. Climate, 5, 252-259, https://doi.org/10.1175/1520-0442(1992) 005<0252:EMASPD $>2.0 . \mathrm{CO} ; 2$.

Woolhiser, D. A., and J. Roldán, 1982: Stochastic daily precipitation models: 2. A comparison of distributions of amounts. Water Resour. Res., 18, 1461-1468, https://doi.org/10.1029/ WR018i005p01461.

Yi, C., 2010: On the first passage time distribution of an OrnsteinUhlenbeck process. Quant. Finance, 10, 957-960, https:// doi.org/10.1080/14697680903373684. 